\title{
Challenges for a Total System Analysis on Deepwater Floating Systems
}

\author{
Subrata Chakrabarti*
}

Offshore Structure Analysis, Inc.13613 Capista Drive, Plainfield, IL 60544, USA

\begin{abstract}
In the design of floating systems, three major system components need special attention: the floater, the mooring system, and the riser system. This paper will focus on one the most unique areas in the structural design of these components, namely, the fluid-induced effects on floating structure systems and the associated structural response. Due to the rapid growth in the offshore field, particularly in deep waters, this analysis is seeing a phenomenological growth, and considerable research is ongoing in this area, with steady advancement in the design procedure. The state-of-the-art in the treatment of the individual components of the floating structure, namely, the floater, the mooring system, and the riser system will be briefly described. The importance of their interactive coupling effects with fluid is the special subject that will be emphasized. In particular, the 'Total System Analysis' of the floating system consisting of all three of its dynamic components will be laid out. A possible systematic approach for the complete system and various simplifications available for an efficient practical solution will be elaborated. The paper will conclude with a discussion of the present-day deep water design challenges that remain and the research that is needed to meet these challenges.
\end{abstract}

\section{INTRODUCTION OF OFFSHORE STRUCTURES}

The design life span of offshore structures ranges from a few years to as many as 25 years based on their applications. Often their useful life extends beyond their design life, and they remain operational longer. In most instances, the floating offshore structures are required to stay in position in all weather conditions. Offshore structures [1] are defined by either their function or their configuration. The functions of an offshore structure may be one of the following (even though multiple functions may be possible for a structure):

- Exploratory Drilling Structures: A Mobile Offshore Drilling Unit [MODU] configuration is largely determined by the variable deck payload and transit speed requirements.

- Production Structures: A production unit can have several functions, e.g. processing, drilling, workover, accommodation, oil storage and riser support.

- Storage Structures: Used in storing the crude oil temporarily at the offshore site before its transportation to the shore for processing.

The configuration of offshore structures may be classified by whether the structure is a fixed structure, either piled or gravity, a compliant or articulated structure, or a floating structure. The requirements of a floating structure are that it be moored in place and that, subject to the environment, the floater remains within a specified circle of operation from a desired mean location, which is generally achieved by mooring lines (or a dynamic positioning system).

\section{FLOATING OFFSHORE SYSTEM}

Floating offshore system [1] illustrated in Fig. (1) consists of three principal structural components:

*Address correspondence to this author at the Offshore Structure Analysis, Inc.13613 Capista Drive, Plainfield, IL 60544, USA; chakrab@uic.edu
- Floating hull: providing the space for the operation of the production work, and the space for storage of supplies,

- Mooring system: providing a connection between the structure and the seafloor for the purposes of securing the structure (generally called station-keeping) against environmental loads, and

- Risers - achieving drilling operation or product transport.

The station-keeping may also be achieved by a dynamic positioning system solely using thrusters, or in combination with mooring lines. The dynamic positioning, however, will not be part of this paper. The mooring lines provide the restoring force to the floater.

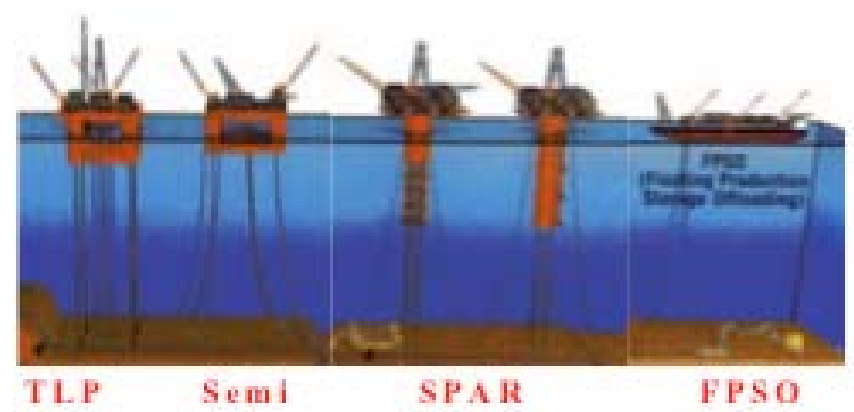

Fig. (1). Floating offshore systems.

\section{TOTAL SYSTEM ANALYSIS}

It appears from the research done to date on the subject of coupled system and the results presented here that, for some systems, the complete coupled system analysis is important, whereas it does not provide appreciably different responses for other floating systems. However, whether such analysis is warranted for a particular system cannot be easily determined a priori at the present time with the present state of knowledge about the systems. More comprehensive re- 
search into such analysis is required to generate such guidelines. Therefore, further systematic research should be made to answer the lingering question regarding the importance of the coupled system analysis [2-20]. The goal should be to generate a region of applications chart for various systems that can be used by the designer as a guide to determine the level of sophistication required in the analysis of a given floating system.

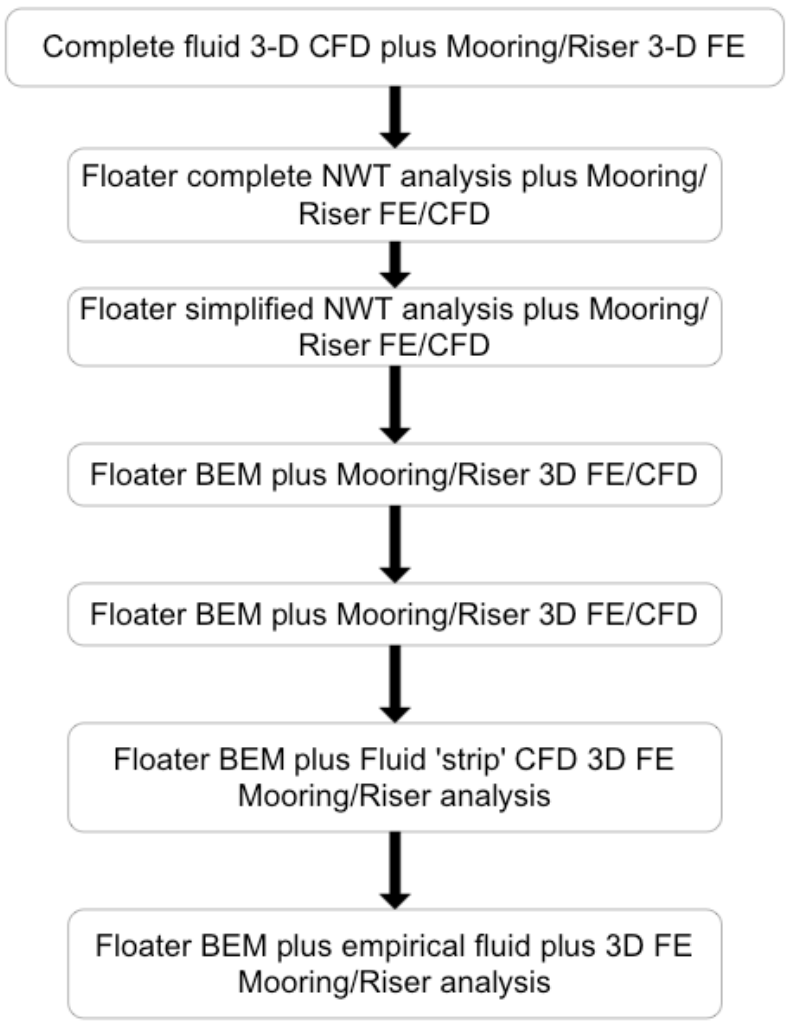

Fig. (2). Sequential approximation from a Total System Analysis (TSA) to a more practical method.

The 'total system analysis' (TSA) is defined here as the analysis of the complete floating system, in which no empirical simplifications are made regarding the loading or the responses of the system components. Thus, the system considers the fluid structure interaction of the entire system, including the coupling among them concurrently. Ideally, it means that both the fluid loading and structure response will include the entire fluid-floater-mooring-riser interaction, (with the incorporation of any soil interaction, as needed) without an ad hoc empiricism. In other words, the fluid flow past the system is represented in a computational fluid dynamics (CFD), while the complete structure system is described in a Finite Element (FE) representation. However, while it is generally possible to incorporate such a complete model in software with the current state of knowledge, in practice, it becomes an insurmountable problem and a prohibitively time-consuming task to accomplish under the present hardware environment. Therefore, the complete analysis must be simplified to achieve a practical limit, but in a logical step with fewer empiricism. These simplifications are proposed here, which may be achieved in a hierarchical sequence, as illustrated in Fig. (2). The sequence is shown starting with the most complete analysis and ending with the simplest and most used analysis adopted today in the design of an offshore system. Thus, any intermediate step in the figure may be considered a refinement in the analysis. Of course, one can come up with a variation of the proposed intermediate steps. But the proposed ones appear logical.

In the complete analysis, the system includes the floater, and all the mooring lines and risers. The fluid field past this complete system is described in a CFD code and the mutual interaction of all the components with the fluid is solved in terms of Navier Stokes equation, in which the floater is represented as a rigid body, but the mooring lines and risers are input in a structural FE code. In the next simplification (step 2 ), the fluid loading on the floater is determined by the more complete numerical wave tank analysis (NWT, to be elaborated later). In this case, the mooring lines and risers are individually analyzed by a 3-D FE analysis, and iteration is performed to achieve convergence. In step 3 of simplification, the floater NWT is approximated before coupling with the mooring/riser finite element (FE) analysis. If further simplification is needed, then the simple 3-D diffraction theory may be used for the floater (step 4). For simplification step 5, instead of full 3-D approach, the appendages (mooring lines and risers) may be handled by a segmented '2-D strip CFD' to represent the 3-D fluid flow field. Finally (in step 6), the risers and mooring lines are solved by a structural finite element analysis or like, in which the fluid loading on them is determined via empirical formulas. This is the most common method of design technique for the offshore system today. For some systems, this simplified technique may become too simplified an approach.

In these cases, one practical method of simplification is to consider that the presence of the floater and other appendages will not influence the fluid loading on the mooring/riser component and vice versa. Thus, for a practical analysis under the present-day hardware platform, the following practical coupled TSA method illustrated in a flow chart in Fig. (3) is recommended. In this method the floater is considered a rigid body for which a nonlinear time domain analysis is invoked. The mooring lines and risers are individually analyzed using CFD method. Even though it is still a timeconsuming process, the larger and faster hardware can handle it today.

For risers, such CFD analysis is being successfully carried out and good progress has been achieved with execution time that is not prohibitively high on available hardware. The method may be extended to the mooring lines with a modest amount of effort. A time domain method will be needed for both the floater and the appendages, so that at each time step, iterations may be performed among their boundaries for convergence, before the next time step is reached. Initially some further simplification may be warranted in the CFD analysis, e.g., strip 2-D for a 3-D CFD.

Additionally, as illustrated in the chart (Fig. 3), these types of mooring line and riser analysis will be able to provide sufficiently accurate hydrodynamic coefficients for risers and mooring geometry for application to the complete coupled system after appropriate validation. Once the coefficients are generated, the simpler empirical methods that are used today can also be applied in a design achieving higher accuracy in the results. The technique, while still computationally time consuming, is possible with today's fast com- 


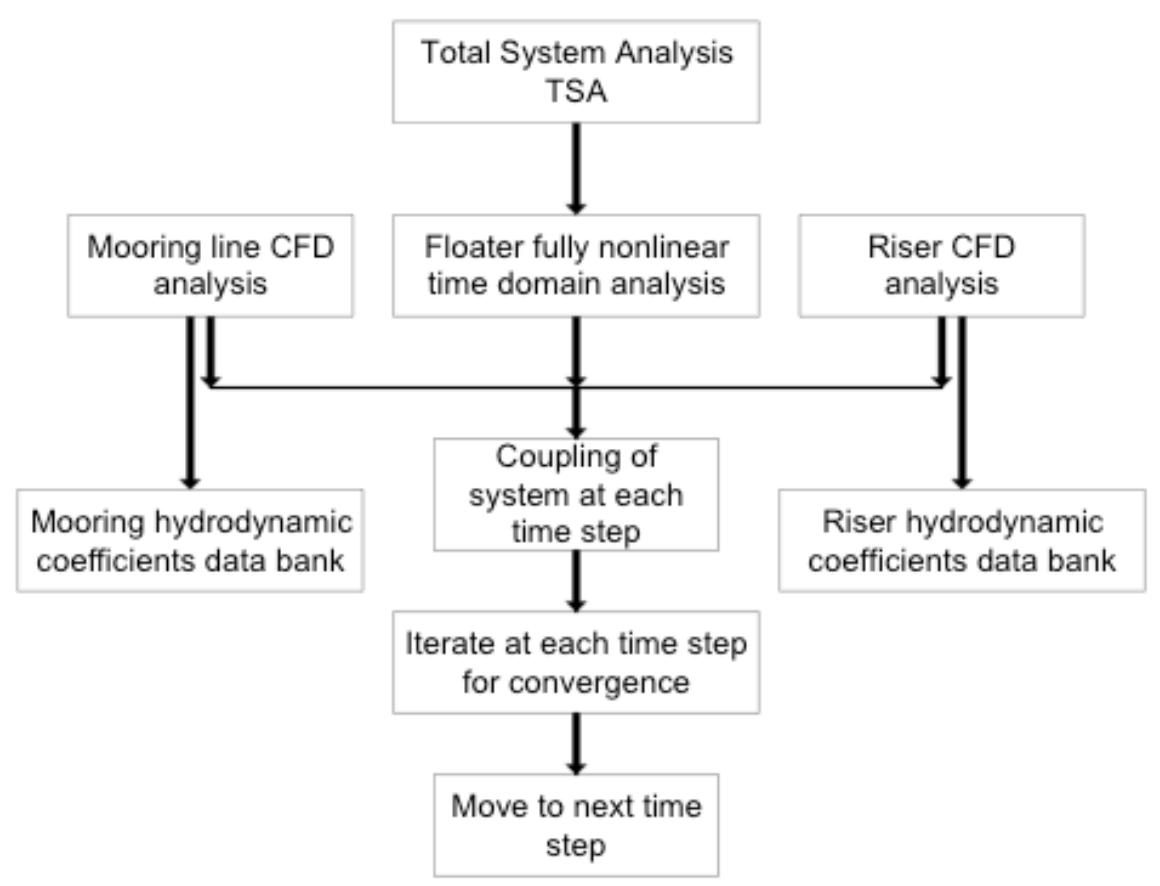

Fig. (3). Flow chart for a Total System Analysis (TSA).

puters and will provide better and more accurate response of the floating system without arbitrarily choosing these coefficients or depending on limited small scale test data. Once such analysis is extensively made, a data bank of coefficients may be generated for future use in the analysis of the coupled system.

In the subsequent sections, the state of the art for the analysis of these systems will be discussed starting with the uncoupled system followed by the individual components.

\section{UNCOUPLED ANALYSIS}

The uncoupled analysis [21-27] considers the traditional methodology, in which the numerical analysis tool is based on the hydrodynamic behavior of the floater, uninfluenced by the nonlinear dynamic behavior of the mooring lines or risers. It is still the common design practice for floating production systems. In the uncoupled analysis procedure, generally few or no integration between the moored system and the risers take place. The uncoupled methodology in design is identified as a distinct two-step procedure as shown in Fig. (4).

\section{Step 1}

The first stage is the design of the floating structure. For the floater, the mooring system is taken as an external nonlinear stiffness term. The stiffness of the riser system is often ignored in this analysis, even though the inclusion of risers, similar to the mooring lines, is generally straightforward without additional complications. In this case, the hydrodynamic model of the floating hull is analyzed for its motion response either in the frequency domain using linear waves or in the more elaborate time domain retaining the nonlinearity in the environment and responses. For the frequency domain analysis, the mooring line (as well as the riser) stiffness is represented by an equivalent overall spring having the slope at the pretension level of the nonlinear stiffness curve (see step 1, Fig. 4). The 6-DOF equations of motion of the floater includes the hydrodynamic inertia, damping, and stiffness terms equated to the forcing function due to waves and current and is solved by a simple matrix inversion. In the time domain, the coupled nonlinear equation of motion is solved by a forward integration scheme.

\section{Step 2}

The second stage of the analysis consists of the design of mooring lines and risers. These components in the floating system are considered individually in their design. The floater motions that result from step 1 (expressed either as RAOs or time series) are applied in three orthogonal components to the top of each individual mooring line and riser at the floater attachment point (see step 2, Fig. 4). In addition, the distributed environmental loads in the form of wave and current loads are applied to the component line or riser. The structural response of the component is computed by employing a lumped mass or a finite-element method.

\section{Floater Dynamics}

The floaters in Fig. (1) that are considered for deepwater exploration and production are mostly large structures. These structures provide considerable obstruction to the incoming waves, and the wave pattern in the vicinity of the floater experiences significant change. Wave forces on large structures are computed by an elegant numerical method on the assumption that the flow past the structure remains essentially potential and the irrotationality assumption for the flow is valid.

\section{Linear Diffraction/Radiation Forces}

The general analytical approach based on linear theory (commonly called the linear diffraction/radiation theory) 

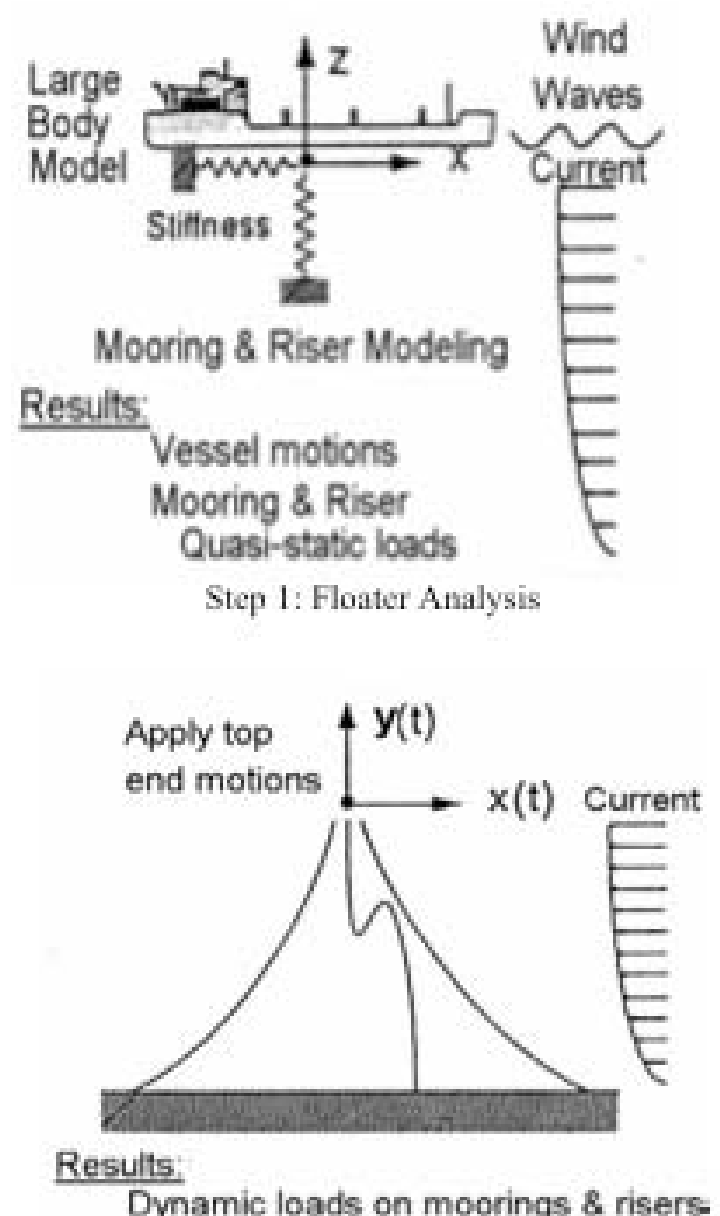

Step 2: Mooring \& Riser Analysis

Fig. (4). Schematic of uncoupled analysis method (adapted from [15].

includes the diffraction and radiation effect from the submerged portion of a structure due to linear progressive waves. In the linear theory, the submerged part of the structure, only under the mean water line (Fig. 5), is considered. Several methods may be used to solve the problem numerically, e.g., fluid finite element method, panel method, hybrid method, etc. [28-48] The most commonly accepted method by the industry is the boundary element panel method (BEM). It makes use of Green's mathematical function and Green's theorem. The spatial part of the total wave potential is written as:

$\phi=\phi_{o}+\phi_{s}+\sum_{n=1}^{6} \phi_{R k}$

in which $\phi_{0}=$ incident potential, $\phi_{\mathrm{s}}=$ scattered potential, and $\phi_{R k}=$ radiated potential due to forced oscillation of unit amplitude in the $k^{\text {th }}$ mode. The incident wave potential at a point $(x, y, z)$ in the fluid is obtained from

$\phi_{0}(x, y, z)=i \frac{g H}{2 \omega} \frac{\cosh k(y+d)}{\cosh k d} \exp (i k x)$

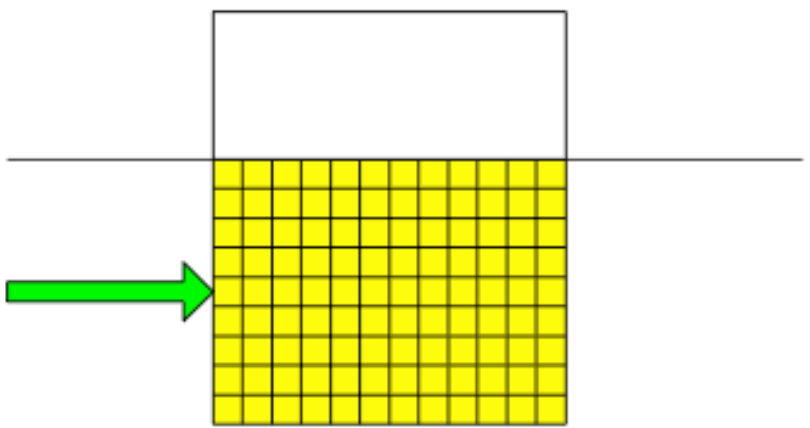

First order force

Fig. (5). Illustrative sketch of the linear force calculation on a floating vertical cylinder.

in which $i=$ imaginary quantity, $g=$ gravitational acceleration; $H=$ wave height; $\omega=$ wave frequency; $k=$ wave number; and $d=$ water depth. In the numerical computation, the submerged surface of the structure is discretized into small flat panels (Fig. 5). The scattered part of the velocity potential in the fluid due to the continuous source distribution on the structure surface is given as

$\phi_{s}(x, y, z)=\frac{1}{4 \pi} \iint_{S} \sigma(a, b, c) G(x, y, z ; a, b, c) d s$

in which $\sigma$ represents the source strength, $(a, b, c)$ represents the source point on the surface of the structure, $(x, y, z)$ the field point in the fluid, and $d s$ is the flat area of the panel on the submerged surface. The function $G(x, y, z ; a, b, c)$ represents the near-field Green's function given in a series or an integral form. The source strength function, $\sigma$, is computed from

$2 \pi \sigma_{s}(x, y, z)-\iint_{S} \sigma_{s}(a, b, c) \frac{\partial G}{\partial n}(x, y, z ; a, b, c) d s=-4 \pi u_{n}(x, y, z)$

where $u_{n}=$ known normal fluid velocity at $(x, y, z)$ due to the incident wave. Equation 4 is solved numerically by setting it up in a matrix form in terms of the centers of the panels. Assuming the field points coinciding with the source points, an $N x N$ complex matrix is formed to describe Eq. 4 where $N$ is the total number of panels on the submerged part of the structure. The solution for the source strengths $\sigma$ at the center of each panel is obtained by the inversion of the complex matrix. For a large value of $N$, this computation is time consuming.

For the radiated potential, the right hand side of Eq. 4 is replaced by the normal unit displacement of the structure at one of six degrees of freedom. Since the only difference here with the diffraction problem is the right hand side, the radiation problem may be solved at the same time, once the inverted matrix is known. The radiated potential provides the added mass and damping coefficients of the structure in 6 degrees of freedom.

Once the diffraction/radiation potentials are known at the center of each panel, the external forces on the submerged body due to the total diffracted (subscript $D$ ) and radiated (subscript $R$ ) potential are obtained respectively from the integrals 


$$
F_{k D}=-i \rho \omega \iint_{S}\left(\phi_{o}+\phi_{s}\right) n_{k} d s
$$

and

$$
F_{k R}=-i \rho \omega \iint_{S_{j}} \phi_{R k} n_{k} d s
$$

where $k=1,2, \ldots 6$ and $S=$ submerged surface area up to SWL (Fig. 5). Thus, the exciting wave forces (and moments) by the linear wave theory are obtained from Eq. 5, while the hydrodynamic added mass and radiation damping are derived from Eq. 6 (see, [49] for details).

\section{Frequency Domain Floater Response}

The environmental forces on the floater are determined at the equilibrium position of the body for a linear analysis. Once the forces on the structure are computed, taken as a rigid body, in a simplest response analysis, the moorings and risers are treated as linear or linearized nonlinear springs, externally attached to the floater at their attachment points.

Moreover, the free motions of the body generate the linear hydrodynamic added mass and damping effect. In particular, the radiated potential from the body produces a $6 \times 6$ force matrix. When nondimensionalized by structure displacements and the oscillation frequency or frequency squared, the radiated forces give rise to a $6 \times 6$ damping coefficient matrix and a $6 \times 6$ added mass coefficient matrix, respectively.

The motions are obtained by solving a coupled set of equations of motion. In a linear analysis, it is even possible to introduce a nonlinear damping in an approximate manner. If a Morison type nonlinear damping is included in a linearized form, the equations of motion become

$$
m_{k} \ddot{x}_{k}+\sum_{l=1}^{6}\left(M_{l k} \ddot{x}_{l}+N_{l k} \dot{x}_{i}+\frac{8}{3 \pi} D_{l k}\left|\dot{x}_{l 0}\right| \dot{x}_{l}+C_{l k} x_{l}\right)=f_{k} ; k=1,2, \ldots 6
$$

where $m_{k}=$ mass or moment of inertia in the $k$-th mode, $x_{k}=$ displacement in the $k$-th direction, dots are time-derivatives, and subscript 0 denotes amplitude, while the variables $M_{l k}$, $N_{l k}, D_{l k}, C_{l k}$, = added mass, linear and nonlinear damping and linear restoring force coefficients respectively due to $l$-th degree of freedom in the $k$-th direction. The restoring force includes the stiffness arising from the structure as well as the mooring lines. The stiffness due to risers are generally ignored in this analysis, but can be easily accommodated in Eq. 7. The stiffness is linear or linearized in a frequencydomain solution. The solutions for 6 DOF motion $x_{m}$ are obtained by the inversion of the $6 \times 6$ matrix on the left-hand side by assuming the motions to be harmonic.

A simple iterative technique is chosen to solve the above linearized equation. In the first step, a linear harmonic solution is obtained assuming $x_{0}=0$ on the left-hand side of Eq. 7. This first iteration value is introduced on the left-hand side in the next step and the process repeated. Generally, 2 to 3 iterations produce convergence.

An example of the computed heave motion of a Truss Pontoon Semisubmersible from Eq. 7 is given in Fig. (6). Note that the TSP has a series of rectangular plates in the bottom structural part of the 4 legs. Different responses shown in the figure refer to the cases with and without the heave plates, which produce additional added mass and additional nonlinear damping in heave.
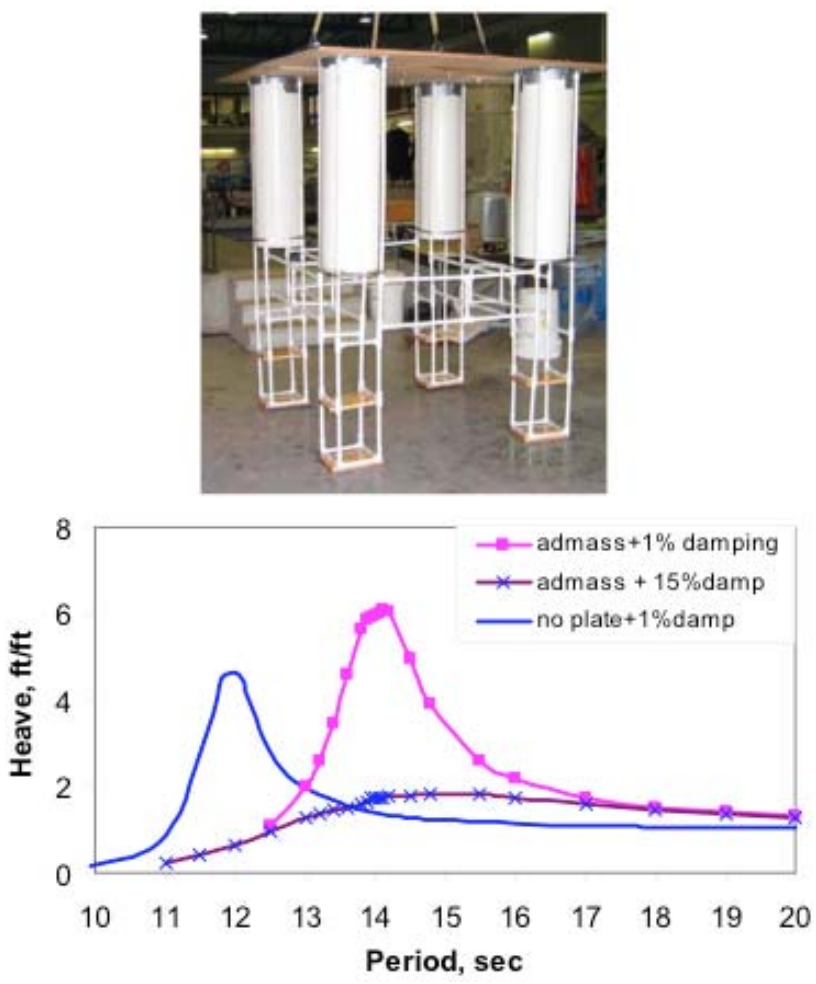

Fig. (6). Example heave response of a Truss Pontoon Semisubmersible including additional heave plate added mass and damping.

\section{Time Domain Approach}

While the above simple linear (or linearized) solution is a useful design tool for a variety of floating offshore structures, it is limited by the linear restoring force, linear (or linearized) damping and linear waves. Some of the nonlinear aspects of the floater motion are well-established, including steady drift force, as well as second-order low frequency (slow drift) and high frequency (TLP tendon) loads.

Approximations for these terms are available that are often used in practice, e.g., Newman's approximation for the steady drift force. While widely used, these approximations are not a significant time-saving computation today. The more complete steady drift force by the pressure-area method is easily computed within the linear diffraction/ radiation program, as this second-order component is derived directly from the first-order potential. The low or high frequency force calculations, however, are much more involved in terms of a quadratic transfer function, which is extremely time-consuming to apply routinely in a design. Therefore, simplified assumptions are often applied to slow drift or high frequency loads using fewer frequency pairs around the resonance frequency to reduce the computation effort. This type of approximation is a common design practice.

In order to account for the nonlinearity properly, a time domain solution is sought. The analysis is still uncoupled and is generally carried out in the time domain assuming the floater to be a rigid body, and the solution is generated by a 
forward integration scheme. The analysis can easily accommodate the nonlinearities present in the excitation forces (e.g., wind, current, steady wave drift, slow drift, etc.), damping (both linear and nonlinear) and restoring forces typically encountered in a moored floating system. The waves can have single frequency or can compose of multiple frequency components following a given spectral model (e.g., PM or JONSWAP). Note, however, that the nonlinearities in the free surface forces are generally only approximated by empirical means in such analyses.

For the series representation of long crested waves for a given wave energy spectrum, the wave profile may be decomposed as:

$\eta(x, t)=\sum_{n=1}^{N} a_{\mathrm{n}} \cos \left[k_{n} x-\omega_{n} t+\varepsilon_{n}\right]$

where $N$ is the number of decomposed wave components and $a_{n}, w_{n}, k_{n}, \varepsilon_{n}$ are the component wave amplitude, frequency, number and random phase respectively. The linear and nonlinear excitation, structure displacement and velocity dependent forces are computed for each of these wave components at each step time. At the given time step, a set of second-order differential equations (similar to Eq. 7, but retaining all the nonlinearities present in the system) is solved to obtain the accelerations of the system. For example, for a single degree of freedom system, the current value of acceleration is computed from the equation of motion for a random force at time $t, F(t)$ as:

$\ddot{x}_{c}=\left(F(t)-c \dot{x}_{p}-k x_{p}\right) / m$

where the subscripts $\mathrm{c}$ and $\mathrm{p}$ stand for the current and previous values respectively. The force time history $F(t)$ may be composed of nonlinear wind and current forces including wind spectrum, the linear diffraction forces, 2 nd order steady and oscillating forces and Morison and lift forces. The solution is initiated with prescribed values for the displacements and velocities, and these values are calculated for the next time step from the derived accelerations by the forward integration scheme, e.g., finite-difference:

$\dot{x}_{c}=\dot{x}_{p}+0.5 *\left(\ddot{x}_{c}+\ddot{x}_{p}\right) * d t$

$x_{c}=x_{p}+0.5 *\left(\dot{x}_{c}+\dot{x}_{p}\right) * d t$

The inclusion of interconnected floating structures in a multi-floater analysis is rather straightforward. The computation is quite efficient and can be accomplished on a mediumsized Personal Computer.

The time-domain analysis has been shown to work quite well in a variety of moored floaters. An example of a time domain solution for a moored floater with multiple mooring lines in intermediate water depth is shown in Fig. (7). The roll and pitch motion of the floater in a strong current and the tension in one of the mooring lines are shown.

\section{Time Domain Fully Nonlinear Forces}

Numerous articles are available on nonlinear wave-body interaction with offshore structures (for example, [30, 37, 38, 48]). Many of these not only consider the nonlinear forces on the floating structure, but the nonlinear response of the structure as well. The consistent nonlinear numerical solutions are
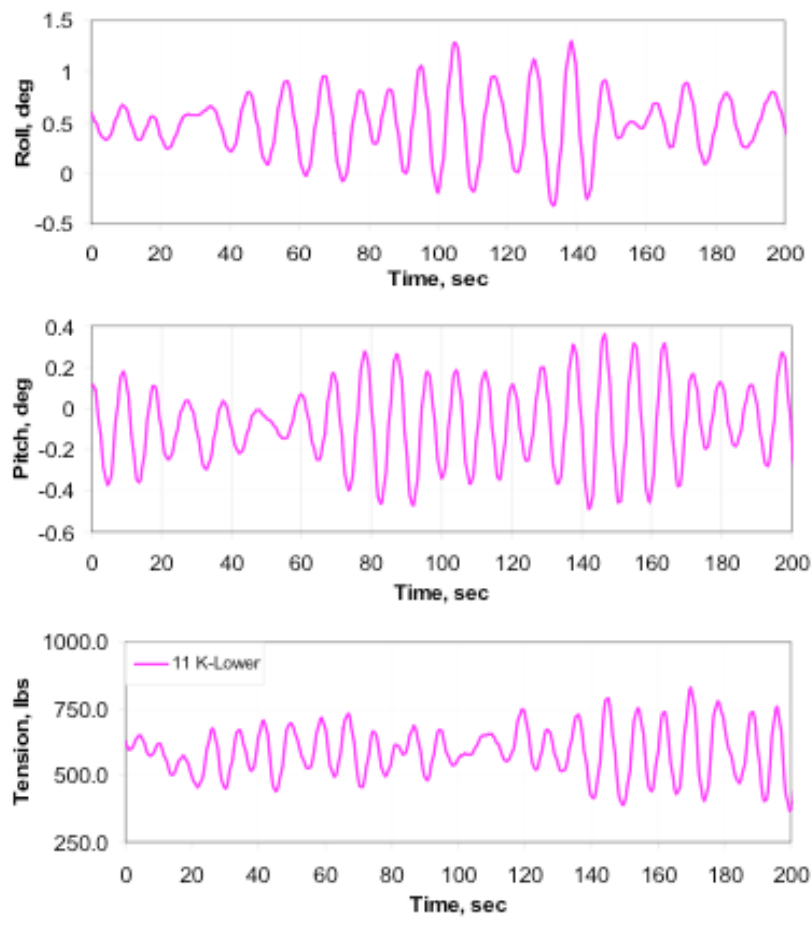

Fig. (7). Time history of motions and load due to a random wave on an SPM.

quite elaborate and extremely time-consuming. The fully nonlinear wave-structure interaction boundary-value problem may be solved by the Mixed Eulerian-Lagragian (MEL) method (see $[28,29,38])$ without any analytical approximations. This method of solution requires prohibitively large computational efforts and is not yet practical for routine industry use. Moreover, several technical issues are yet to be satisfactorily resolved before this approach can be successfully applied for complex 3-D offshore structures [27]. To address the need of the industry, several time-domain solution methods have been proposed, which minimize this excessive use of computational efforts, but account for the essential nonlinearities in approximate ways (see, for example, $[24,5,27])$. In most cases, the hydrodynamic interaction due to radiation and diffraction effects is linearized. This allows the use of the usual 2-D or 3-D linear diffraction/radiation theory. Considerable development in the area of Numerical Wave Tanks (NWT) has been made in the last decade [47, 50]. A review article [51] summarizes some of these works. They have been applied to a variety of simulations like fully nonlinear free-surface waves, wave radiation by forced oscillated body (radiation problem), wave and fixed body interaction (diffraction problem) and floating body dynamics (radiation and diffraction problem). In order to apply NWT to the design of offshore structures, simulated hydrodynamic forces must be accurate and reliable.

Here a 2-D nonlinear wave - fixed structure interaction problem is formulated using a potential-based fully nonlinear NWT. Fig. (6) shows the definition sketch for a vertical cylinder assumed frozen at an instant in wave (thick line). The theory is based (closely following the work of Kim and Koo [40]) on mode-decomposition, and Mixed EulerianLagrangian (MEL) material-node time marching scheme (e.g. [42]), and uses the boundary element method (BEM). 


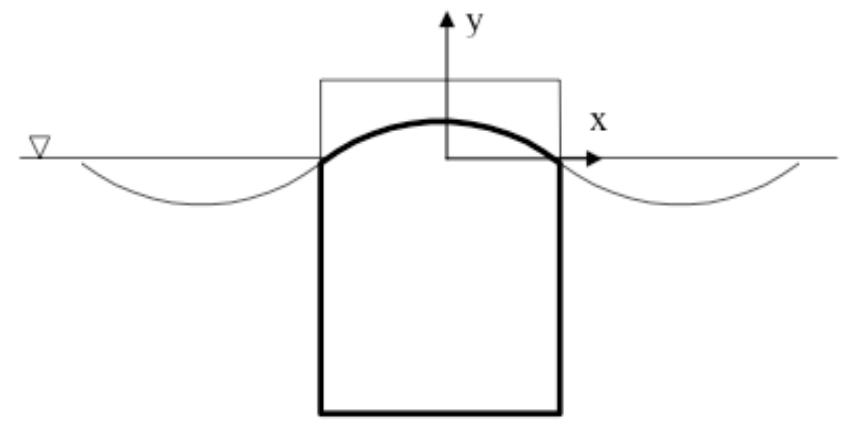

Fig. (8). Illustrative sketch of the fully nonlinear force calculation on a fixed vertical cylinder.

As in the case of linear diffraction/radiation theory, an ideal fluid is assumed so that the fluid velocity can be described by the gradient of velocity potential $\boldsymbol{\Phi}$. A Cartesian coordinate system is chosen such that $y$ is positive upwards corresponding to the still water level. Then, the governing equation of the velocity potential is given by

$\nabla^{2} \Phi=0$

The boundary conditions consist of

$>$ Fully nonlinear dynamic free surface condition, satisfied on the exact free surface (thick line in Fig. 8):

$\frac{\partial \Phi}{\partial t}=-g \eta-\frac{1}{2}-|\nabla \phi|^{2}$

$>$ Fully nonlinear kinematic free surface condition, satisfied on the exact free surface:

$\frac{\partial \eta}{\partial t}=\frac{\partial \Phi}{\partial y}-\nabla \phi . \nabla \eta$

$>$ Body boundary condition on the structure:

$\frac{\partial \Phi}{\partial n}=0$

$>$ Inflow boundary condition: At the inflow boundary, a theoretical particle velocity profile is fed along the vertical input boundary. The exact velocity profile of a truly nonlinear wave under the given condition is not known a priori. Therefore, the best theoretical wave profile is input. Since the fully nonlinear free-surface condition is applied in the computational domain, the input wave immediately takes the feature of fully nonlinear waves. Any unnecessary spurious waves inside the domain is accounted for and corrected.

To simulate the open-sea condition, a proper outgoing wave condition at the radiation boundary needs to be imposed. The most physically plausible open boundary condition is Sommerfeld/Orlanski outgoing wave condition [44]. The Orlanski radiation condition, for example, was used by [30] for the simulation of nonlinear regular and irregular waves and by Isaacson and Cheung [35] and Kim and Kim [39] for wave-current-body interaction problems. There exists other open-boundary conditions, such as absorbing beaches by artificial damping on the free surface ([28, 29, $31,33,41]$ etc.) or matching with linear time-domain solutions at far field [32]. A properly designed artificial damping on the free surface need not be far from the body and can damp out most wave energy, if its' length is greater than two wave lengths. Therefore, the Orlanski condition is considered more effective than the matching technique and is ideal in damping out relatively short waves [40]. The artificial wave absorbing damping zone (Fig. 9) is introduced in the free surface boundary conditions.

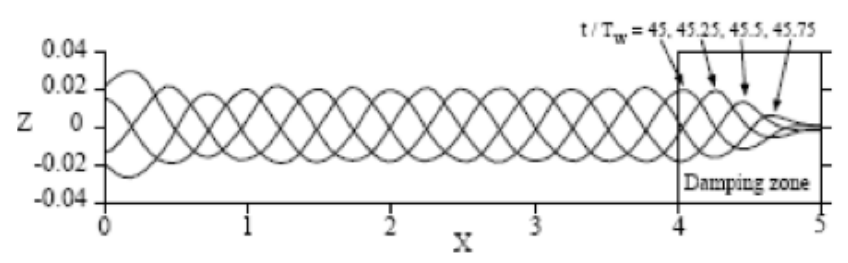

Fig. (9). Dissipation of waves in the artificial damping zone [51].

An integral equation in terms of two-dimensional Green function satisfying Laplace equation is adopted. To update the fully nonlinear kinematic and dynamic free-surface conditions at each time step, Runge-Kutta $4^{\text {th }}$-order timeintegration scheme [23] and the Mixed Eulerian-Lagrangian approach are adopted. Lagrangian approach for which the free-surface nodes move with the water particle is used. At each time step, (i) the Laplace equation is solved in the Eulerian frame, and (ii) the moving boundary points and values are updated in a Lagrangian manner. To avoid non-physical saw-tooth instability on the free surface in the time marching scheme, smoothing and regriding methods are used. In the case of fully Lagrangian approach, the free-surface nodes need to be updated and rearranged at every time step. The regriding scheme prevents the free-surface nodes from crossing or piling up on the free surface, and thus makes the integration more stable.

When the simulation is started, a ramp-function [23] at the input boundary may be applied. The ramp function prevents any impulse-like behavior and consequently reduces the corresponding unnecessary transient waves. As a result, the simulation is more stable and soon reaches the steady state. Towards the end of the computational domain, an artificial damping zone is applied on the free surface so that the wave energy is gradually dissipated in the direction of wave propagation. The profile and magnitude of the artificial damping is designed to minimize possible wave reflection at the entrance of the damping zone, while maximizing the wave energy dissipation.

Accurate calculation of the time derivative of velocity potential is very crucial in obtaining correct pressure and force on the body surface at each time step. There are several ways to achieve it. Backward difference is the simplest way using the potential values of previous time steps. In case of a stationary structure, more accurate finite-difference formulas [23] can also be used. The wave force on the body surface is calculated by integrating Bernoulli's pressure over the instantaneous wetted surface from the nonlinear wave. While the above development is shown for a wave force computation, the method can be extended to include moving structures as well (see [40]).

\section{MOORING SYSTEM}

During the preliminary design and analysis of the floating structure response, as stated, the mooring lines are suc- 
cinctly assumed to be merely nonlinear springs and the effects of the environment on the lines themselves are ignored, on the assumption that their overall effect on the floater response is small. In the design of these lines themselves, however, the environmental forces may be an important criterion and may need to be considered in the final design analysis [52-59].

In the process of designing and developing an offshore system with mooring lines, one first selects the lines' layout, geometry, and the initial mechanical and structural properties. Different types of mooring and anchoring methods for today's floating offshore systems are shown in Fig. (10). The lines (typically 8 to 12 ) are arranged in a symmetric arrangement, except to make room for risers, if needed. There are principally two types of mooring systems in use today catenary (or, semi-taut) mooring and taut mooring system. The catenary lines often consist of chain-wire-chain combination. The taut lines could be steel wires or synthetic polyester. It is customary to use drag anchors for the catenary lines. For the taut polyester lines, a vertically loaded plate or a suction anchor (Fig. 10) may also be suitable.

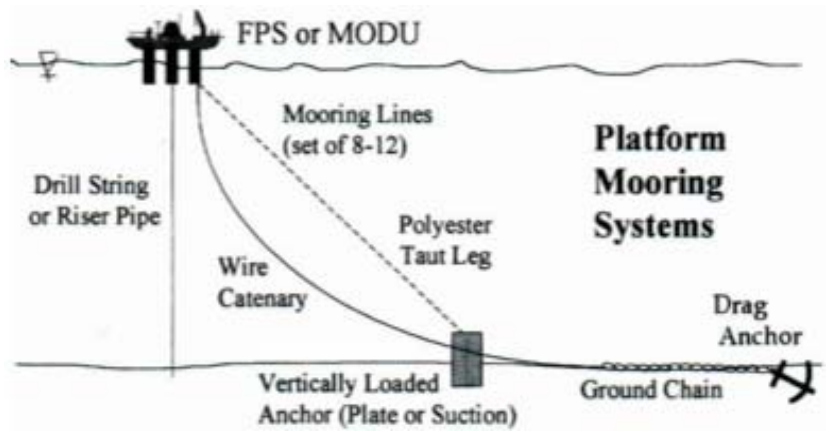

Fig. (10). Schematic layout of mooring and anchoring system.

For the taut mooring system the restoring force comes primarily from the line stretch. The restoring force for the conventional catenary system results, primarily, from their submerged weight and changes in catenary shapes, as the line displaces with the floater due to environmental loading.

\section{Design of Mooring Lines}

Fig. (11) shows a catenary mooring line spread between the anchor point on the ground and the fairlead point at the structure subjected to the environmental loads. A portion of the line lies on the ground. The line will lift off the touchdown point under loads and will take a different catenary shape giving an increase in the tension at the fairlead point. The design of a mooring line is performed in several ways depending on the design stage and the sophistication required in the analysis. Quite often the design is static, in which the load characteristics for a single line as well as the mooring spread are established from the horizontal excursion of the line ignoring dynamic environmental loads on the line itself. The static mooring design analysis employs the following steps.

The load-elongation characteristics are first computed for each line, given the line end-point coordinates, lengths and elasticity. The forces for all lines in the mooring spread are then summed based on their orientation to yield the resultant horizontal and vertical restoring force vs. displacement of the vessel. The overall mean offset of the vessel caused by only the steady loads from wind, current, and wave drift is estimated. The restoring force and tension in the most loaded line are computed by displacing the vessel through this displacement. The safety factor for the most heavily loaded line is computed based on the breaking strength of the line. If it is too low, some of the selected design parameters, e.g., line pre-tension, material specifications, the end co-ordinates or the number of lines, are adjusted and the above calculations are repeated. The design is checked again with the balance of the lines, assuming that the most loaded line is broken.

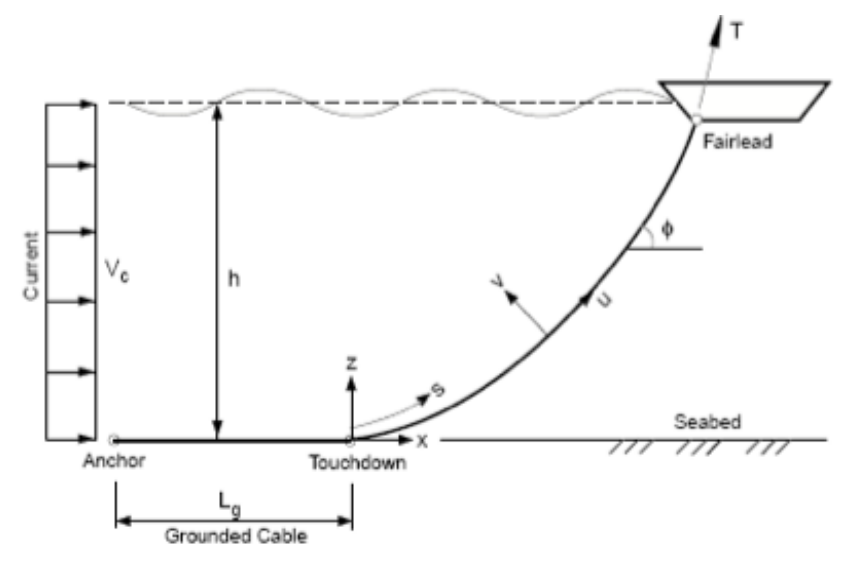

Fig. (11). Schematic of a typical mooring line under load [59].

In a quasi-static design, the line dynamics are still ignored, but the dynamic loads on the floater are included in the analysis as a quasi-static load. Thus, in addition to the vessel offset from the mean wind, current and wave drift forces on the vessel, the maximum excursion from the wave induced vessel motion at wave and slow drift frequencies are computed. Then the maximum line tensions resulting from the total vessel offset for each possible environmental direction are determined. The line tensions with a safety factor (generally taken as 2) are compared with the minimum breaking load. DNV currently suggests separate safety factors for the mean load and the dynamic load amplitude to avoid excessive conservatism, since mean loads are much higher and more reliably predictable. As before, the maximum peak line loads with one broken line are recalculated. If the proposed mooring specification fails the safety factor test, then a new specification is tried.

The dynamic design of the mooring lines includes additional loads on the mooring lines themselves. In an uncoupled analysis, the motions of the platform independent of the line dynamics are firstly calculated to determine the top-end oscillation of lines (Fig. 4, step1). The external loads on the floater in this part include steady and fluctuating wind, wave and wave drift, and current. In the second part (Fig. 4, step 2 ), the mooring line dynamics are determined, for which a time domain analysis is required. Loads and responses on the mooring lines include XYZ motions of the floater at the fairlead point, current, and wave loads on the line, and the seabed lateral friction on the grounded portion of the line, including soil spring and soil damping.

In discretizing the mooring lines, either a finite element model, e.g., [55] or a lumped mass approach [56, 57] may be employed. The lumped mass method is more straight for- 
ward and more efficient. In a lumped mass method, the line is de-composed into a number of straight elements (bars) with linear shape function (Fig. 12). The distributed mass plus added mass is lumped at the end nodes.

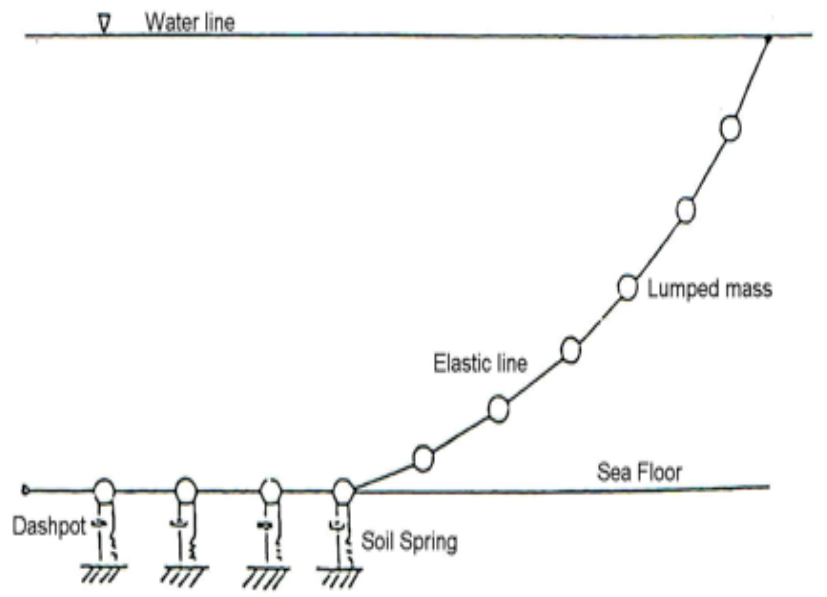

Fig. (12). Numerical analysis model for dynamic effect on a mooring line (adapted from Inoue and Surendran [56]).

The hydrodynamic damping term includes the relative motion between the line and the fluid and a damping coefficient. Damping levels may vary significantly depending on water depth, soil properties for grounded cable (Fig. 12), line make up, offsets and top-end excitation. Similarly, the inertial effect between the line and the fluid may be included (though this influence is often small). At each time step, a standard set of matrix equation is developed composed of the inertia, damping and stiffness matrix at the lumped masses. The solution is obtained by the inversion of this matrix equation.

In order to study the effect of the seabed interaction, Ong and Pellegrino [59] performed a parametric study with two chosen lines: a $0.14 \mathrm{~m}$ diameter chain (Cable 6) in shallow waters and a $0.13 \mathrm{~m}$ diameter cable (Cable 7) in deep waters. Six different configurations (A-F) shown on the left hand side of Fig. (13) were chosen.

The results obtained from quasi-static, frequency and time domain analyses were compared. The cables were excited in still water at the fairlead by a horizontal excitation of $1 \mathrm{~m}$ over wave periods of $4 \mathrm{~s}$ to $40 \mathrm{~s}$. For Cable 6 , the dynamic amplification was found to be relatively insensitive to the configurations. Fig. (13) shows the dynamic amplifications of fairlead tension for Cable 7 for different models. Significant reduction in tensions can be seen in Models A, D and E where seabed interaction is included. Note that there is no dynamic amplification for Model $\mathrm{F}$, since it is based purely on a static calculation. For both cables, catenary action appears to be less important than stretching of the grounded cable. The effect of catenary action became more prominent (Fig. 14) when the amplitude of excitation increased up to $10 \mathrm{~m}$.

It is often important to check the fatigue life of the mooring line. In this case, a mooring line fatigue analysis may be based on long term cycle of dynamic tension due to time varying wave forces. In fatigue, the recommended factor of safety (FOS) is between 3 and 10 depending on the lines
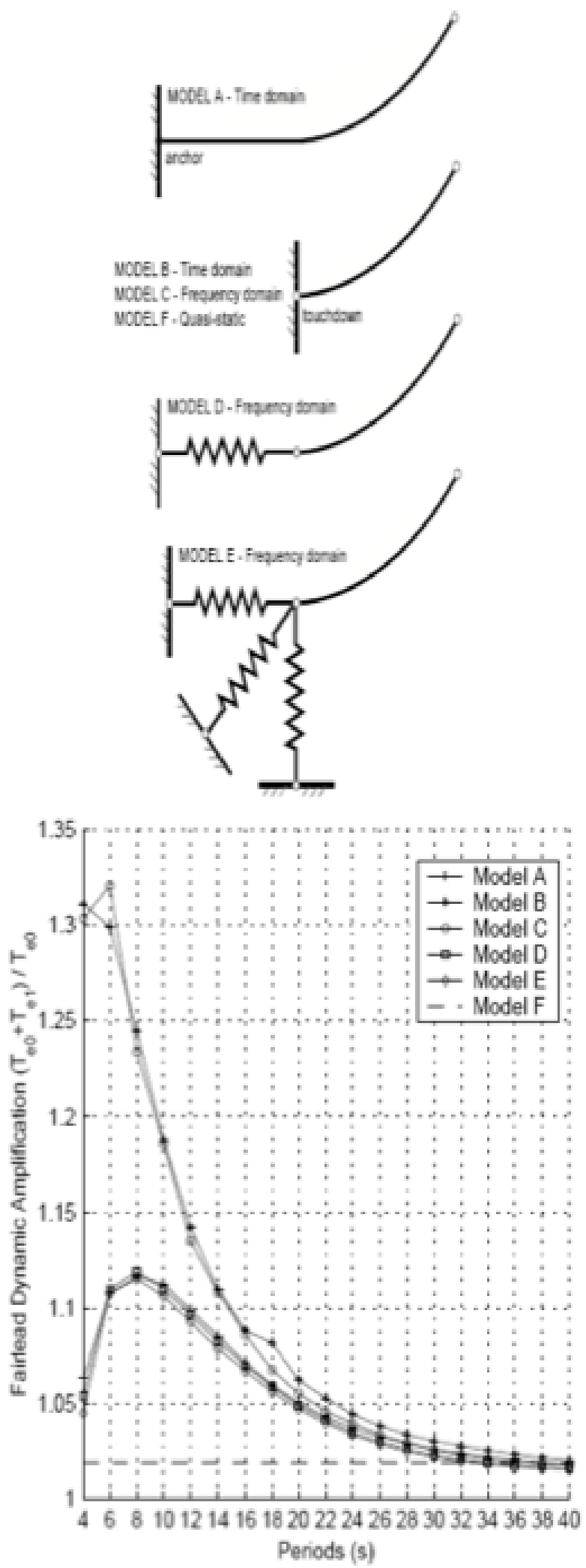

Fig. (13). (a) Cable configurations and (b) Fairlead dynamic amplification of cable no.7 [59].

being inspectable or not. Because of the low level of experience of lighter polyester lines in deepwater application, today the FOS for a polyester line is taken to be high, as much as 60 . 


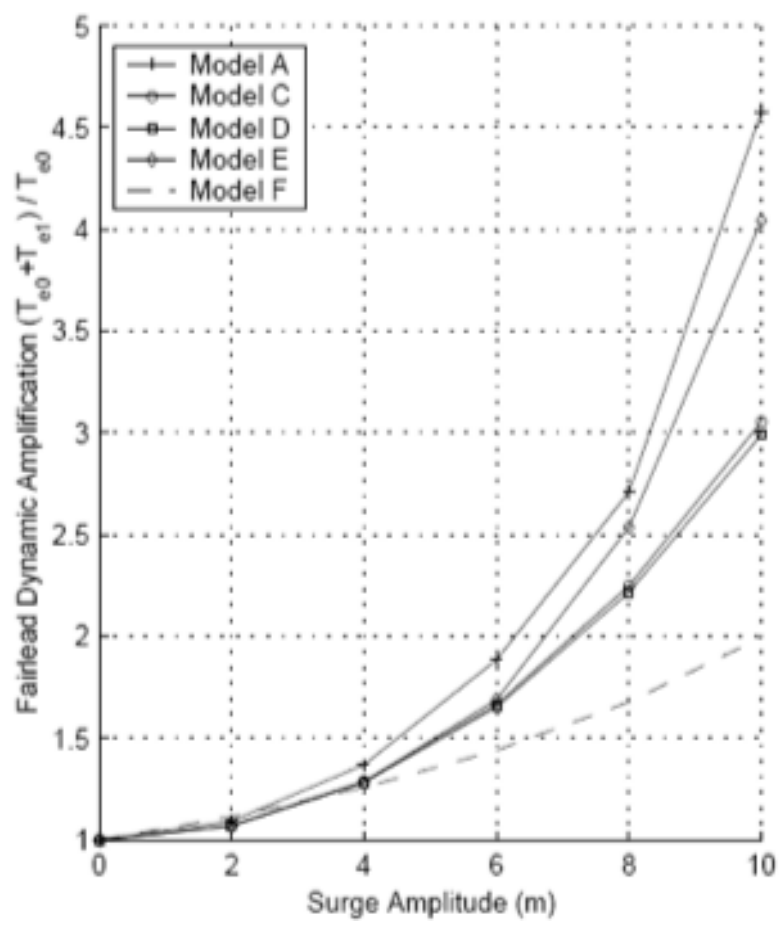

Fig. (14). Dynamic amplification at the mooring fairlead vs. floater surge amplitude for a 10 s period [59].

\section{RISER SYSTEM}

As noted earlier, the riser is an important component of an offshore structure and, for floating structures, there are a variety of risers in use today. They are schematically identified in Fig. (15). The vertical risers are still most common and used for the drilling operation. These risers for drilling and production operations are pre-tensioned (Top Tension Risers, TTR). The production risers may have a catenary shape (Steel Catenary Riser, SCR) or may be flexible as well. Flexible risers may have additional buoyancy elements giving it an $\mathrm{S}$-shape.

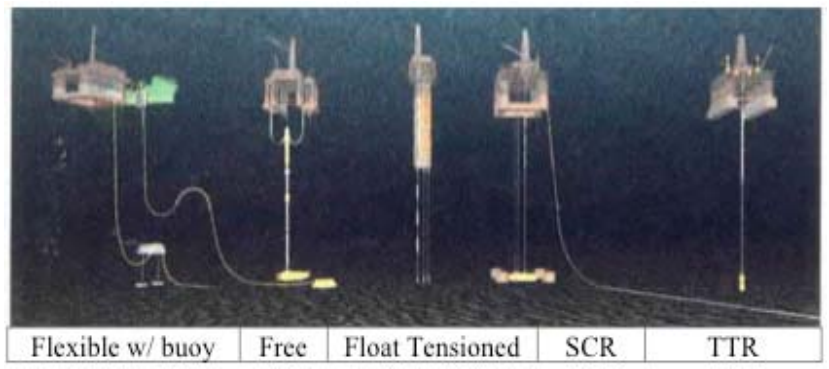

Fig. (15). Typical risers in use today with a variety of floaters.

\section{Dynamics of Risers}

Today almost all practical riser design analyses are performed by empirical methods. Static analysis is generally based on steady loads and provides the riser deflections, top and bottom angles, mode shapes, and riser stresses. Dynamic analysis considers the inline and transverse unsteady loads. In addition to the riser shapes and stresses, it provides the vortex induced vibration, and fatigue life of the riser.
In order to implement more advanced design of risers [61-77] in practice, the current development of riser technology is advancing the coupled riser FE/CFD numerical analysis, in parallel with small-scale laboratory, and larger scale or full-scale in-situ testing. In addition, several commercially available large CFD programs are updated to make them more suitable for offshore applications. Today, the limitations of such analyses, in part, has been large computational time, limitations in flow solver (including convergence), and high Reynolds number. They also need systematic validity with reliable benchmark tests, which are in most part still lacking. The laboratory testing is generally performed at the water channels, and wave tanks at a University research laboratory. They are sometimes limited by small scales, modeling problems, and accurate measurements. The principal purpose of these tests is to increase the understanding of the fluid flow problems past risers under various conditions, as well as to generate empirical coefficients. The advantage of such testing is the control of various independent parameters that influence riser dynamics in a systematic way. Field measurements often include in-situ testing in full scale and face the constraints of cost, environment, practicality, and confidentiality.

\section{Mathematical Model}

In this section a basic mathematical model for the riser analysis is developed. It should, however, be recognized that there are many approaches in the dynamic analysis of risers and their more comprehensive numerical details are considered outside the scope of this paper. Therefore, the development below should be taken as an exercise to introduce the basic parameters and the governing equations for the riser dynamics.

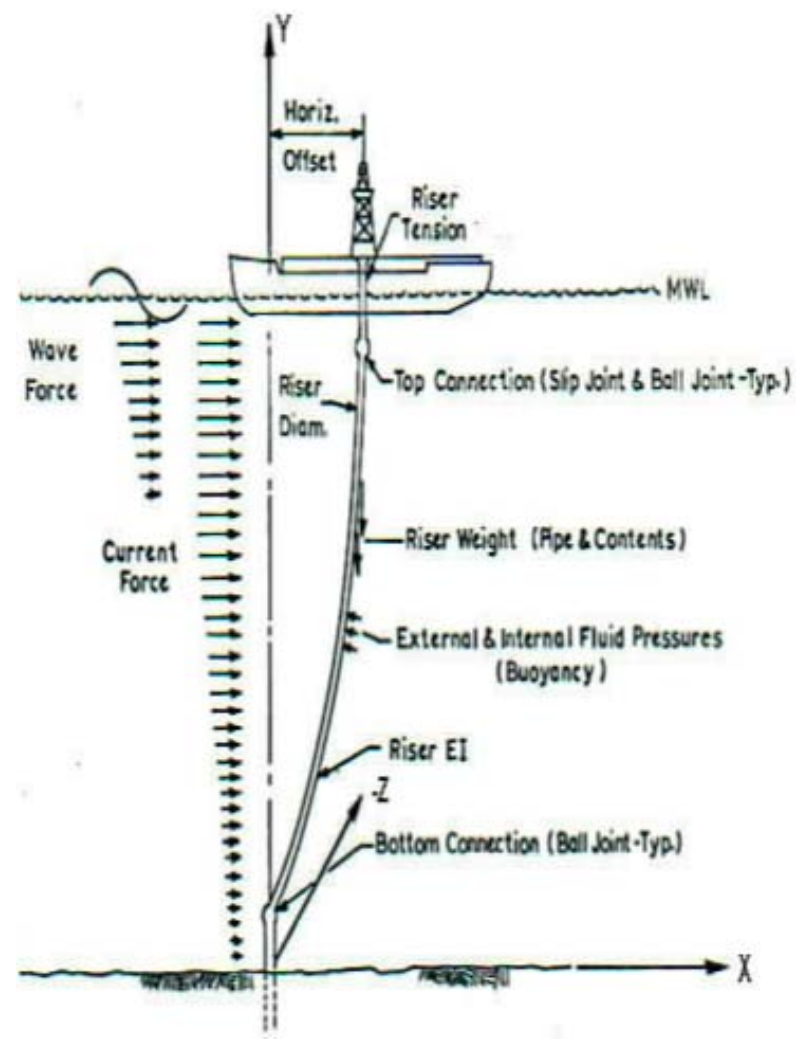

Fig. (16). Schematic of vertical top-tension riser in current. 
Consider a vertical top-tensioned riser exposed to ocean waves and currents, as illustrated in Fig. (16), in which the waves and current are shown as collinear. The definition of the co-ordinate system is shown in the figure. The riser is shown attached to a floater at top and near the ocean floor with appropriate joints which allow rotations of the riser. These risers are generally pre-tensioned (TTR). The upper end is constrained to the floater through the top tensioner, but free to move with the floater both in the horizontal and vertical directions. The various component forces on the riser are indicated in the figure. Considering only current loads acting on the riser, the motion of the riser is governed by the following equations.

For the static analysis of the riser in the inline direction, the right hand side of the equation represents the current force:

$$
\begin{aligned}
& \frac{d^{2}}{d y^{2}}\left(E I(y) \frac{d^{2} x}{d y^{2}}\right)-\frac{d}{d y}\left[F_{e}(y) \frac{d x}{d y}\right]+m(y) \ddot{x}+c(y) \dot{x} \\
& =\frac{1}{2} \rho C_{D}(y) D(y) U^{2}(y)
\end{aligned}
$$

where $x=$ direction of current, $y=$ vertical direction, $m=$ total mass (including hydrodynamic added mass) per unit length of riser section, $c=$ damping coefficient of riser section, $\rho=$ mass density of water, $F_{e}(y)=$ effective tension due to axial tension as well as internal and external fluid pressure forces, $U(y)=$ current velocity as a function of the vertical coordinate $y$, and $C_{D}(y)=$ drag coefficient along the riser.

For transverse riser analysis, the right hand side is the transverse (or lift) force in a static sense:

$$
\begin{aligned}
& \frac{d^{2}}{d y^{2}}\left(E I(y) \frac{d^{2} z}{d y^{2}}\right)-\frac{d}{d y}\left[F_{e}(y) \frac{d z}{d y}\right]+m(y) \ddot{z}+c(y) \dot{z} \\
& =\frac{1}{2} \rho C_{L}(y) D(y) U^{2}(y)
\end{aligned}
$$

where $z=$ transverse direction, and $C_{L}(y)=$ maximum lift coefficient for the riser. It is assumed that the riser bottom end is connected to a frictionless ball joint. Note that the transverse (or lift) force is oscillatory and, therefore, a dynamic analysis will be more appropriate for it.

For a mathematical solution of the system governed by the above equations, suitable initial conditions are also needed. For example, the initial conditions may be given by the static solutions with zero initial velocities. The standard modal superposition method may be used to solve the problem. Alternatively, a finite element model may be applied.

For the dynamic riser solution in waves, the right-hand sides of Eqs. 16-17 are replaced by the forces due to wave. In this case the modified Morison equation may be used for the inline direction (Eq. 16), and the appropriate form of the oscillatory lift force is used in the transverse direction (Eq. 17). The static and dynamic loads may also be combined in a single analysis, even though it is rarely found in the literature.

There are several empirical codes to analyze the vortexinduced vibration (VIV) problem, most of which analyze only the cross-flow (i.e., transverse) response in current. A few of these applications are SHEAR7 (MIT), VIVA (MIT),
VIVANA (MARINTEK/NTNU), VICoMo (NTNU), and ABAVIV (Technip). Some of them also perform the in-line static deflection using amplified $\mathrm{C}_{\mathrm{D}}$ values from the crossflow oscillation, in order to take into account the increase in the drag due to riser motion.

\section{CFD Numerical Model}

While the empirical method of riser design is the current accepted method, considerable efforts are being spent in the more elegant numerical approach. The numerical analysis considers the turbulent incompressible Navier-Stokes equations describing the conservation of mass and momentum in three-dimensions. In this case, a CFD type analysis incorporating the fluid and the structure is desirable. Due to high Reynolds number encountered in such problems, the CFD analysis is quite involved and time-consuming. However, the recent results look promising [68].

At high Reynolds number, there exist a number of different size vortical structures. If the small ones are not modeled correctly, the computed energy transfer from large-scale to small-scale will be incorrect, and the large vortices will be in error. This is a major problem with the current state of turbulence modeling in CFD. This, in reality, involves two problems: (1) having the correct (or a good) turbulence model (i.e., the proper mathematical model), and (2) accurately and efficiently solving the mathematical model. The finite element analysis approach, using the present formulation provides the most accurate practical solution method. The stabilizing operators do not damp out the solution for robustness. It only adds enough and in the direction needed to counteract the destabilizing effects of the convective and divergence free terms. Regarding the first item, this is still an open issue.

\section{Mathematical Model}

The following transient turbulent incompressible NavierStokes equations are considered in a CFD numerical analysis [64]. They describe, respectively, the conservation of mass and momentum in three-dimensions:

$\nabla . \vec{u}=0$

$\rho \frac{D u}{D t}=-\nabla p+\nabla . \tau$

where $\nabla=$ operator, $\vec{u}=$ velocity vector; $\rho=$ fluid density; and $p=$ fluid pressure. The left hand term of Eq. 19 is the material derivative; and $\tau$ is the stress tensor, which models the total contributions from the viscous and turbulence Reynolds stress,

$$
\tau=\left(\mu+\mu_{t}\right)\left(\nabla u+\nabla^{T} u\right)
$$

where $\mu$ and $\mu_{\mathrm{t}}$ are the molecular and turbulent eddy viscosities, respectively and the superscript $T$ represents the transpose. The one-equation RANS turbulence model [76] represents the eddy-viscosity with the Detached-Eddy Simulation (DES) modification. This model is written as

$$
\frac{D \tilde{v}}{D t}=c_{b 1} \tilde{S} \tilde{v}+\frac{1}{\sigma}\left(\nabla \cdot(v+\tilde{v}) \nabla \tilde{v}+c_{b 2}(\nabla \tilde{v})^{2}\right)-c_{w 1} f_{w}\left(\frac{\tilde{v}}{d}\right)^{2}
$$

where the quantity $\tilde{v}$ is the unknown field. The definition of $\tilde{S}$ and $f_{w}$ and constants $c_{b 1}=0: 1355 ;=2 / 3 ; c_{b 2}=0: 622 ; c_{w 1}$ 
$=c_{b 1} / \kappa^{2}+1-c_{b 2} / \sigma, \kappa=0.41$ [77]. The quantity $\tilde{v}$ is closely related to the kinematic eddy viscosity. The turbulence eddy viscosity is given by

$\mu_{t}=\rho \tilde{v} \frac{\chi^{3}}{\chi^{3}+c_{v 1}^{3}} \quad \chi=\rho \tilde{v} / \mu$

The above equations constitute a system of five equations with five unknowns. These equations are solved using the Galerkin/Least-Squares finite element technique (see [73] and references therein for an in-depth description). Equalorder nodal interpolations for all working variables, including pressure, are used with low-order elements. Moreover, the semi-discrete generalized- $\alpha$ method of Chung and Hulbert [69] is used to resolve the time dependency. The Galerkin finite element formulation provides the base algorithm. This formulation, which is equivalent to the central difference formulation in a finite difference method, does not, however, yield stable discretization for the solution of the incompressible Navier-Stokes equations. The difficulties in the stability arise from two main sources: (1) the divergence-free constraint, i.e., the continuity equation; and (2) the convective term in the momentum equations. The leastsquares operator is designed to add the needed stability without sacrificing accuracy.

If the above numerical method is applied to a marine riser in deep water, one of the fundamental problems in the simulation of the long riser is the large size of the CFD problem itself [68]. For a riser in several thousand meters of water depth, the use of three dimensional CFD solutions is impractical by today's computing capabilities. Therefore, a more practical approach to riser response analysis in fluid is to divide up the riser into smaller segments along its axis and to apply a series of 2-D fluid flow solutions within these segments. These 2-D fluid "strips" are combined with the 3D structural model of the riser in a practical way to predict the riser response. In order words, the trick is to reduce the large 3-D CFD problem to a large number of smaller 2-D fluid structure interaction problems. However, this "strip" method has some serious shortcomings. In particular, the flow around bluff bodies, e.g., a riser, is inherently three dimensional so that the 2-D strip solutions can only be expected to be approximate. In addition, the flow is often at a steep angle to the riser, such as, steel catenary risers (SCRs) creating strong axial flow components. Also, VIV suppression devices like helical strakes that have a very strong three dimensional wake cannot be modeled well by the 2-D approximation. Finally, the strip method requires an interpolation method to estimate the forces across the adjacent strips. There is currently no general rules available to make such interpolations [68].

Additionally, other difficulties remain, as explained by Constantinides, et al. [68]. Although the nonlinear material response is small for the riser, nonlinear geometric effects, such as displacements and rotations, are quite large. Thus, a nonlinear structural model must eventually be incorporated in the numerical solution. Also, large motions of the generated meshes must be accommodated and the flow speed and direction are expected to change over the entire length of the riser. A general solution must be able to treat these effects.
Constantinides, et al. [68] used a linear structural model including changes in the current speed and direction with depth. The risers are modeled as tension members and the bending stiffness of the riser is considered negligible compared to the stiffness due to tension. If the eigenmodes are assumed sinusoidal, then the eigenvectors have the form:

$$
\zeta_{i}^{n}=\sin \left(\frac{n \pi y}{L}\right)
$$

where the $\zeta_{i}^{n}$ is the eigenvector associated with the nth mode and the index $i$ indicates the orthogonal $x$ or $z$ directions across the riser axis, $n$ is the mode number, $L$ is the riser length, and $y$ is the distance along the riser axis. The use of a sine shape in Eq. 23 implies that the riser tension is constant along the riser length. With this approach, the motion of the riser is assumed to be a linear superposition of the various eigenmodes. The riser response is computed from the matrix equation:

$\left[m_{i}^{n}\right]\left\{\ddot{y}_{i}^{n}\right\}+\left[c_{i}^{n}\right]\left\{\dot{y}_{i}^{n}\right\}+\left[k_{i}^{n}\right]\left\{y_{i}^{n}\right\}=f_{i}^{n}$

where $y_{i}^{n}$ are the modal amplitudes, and $m_{i}^{n} \quad m_{i}^{n} \quad k_{i}^{n}$ are the associated mass, linear damping and stiffness for each mode. The forces $f_{i}^{n}$ are computed at each time step using the corresponding eigenvector and the displacement of the riser for the particular mode is then computed before moving to the next time step.
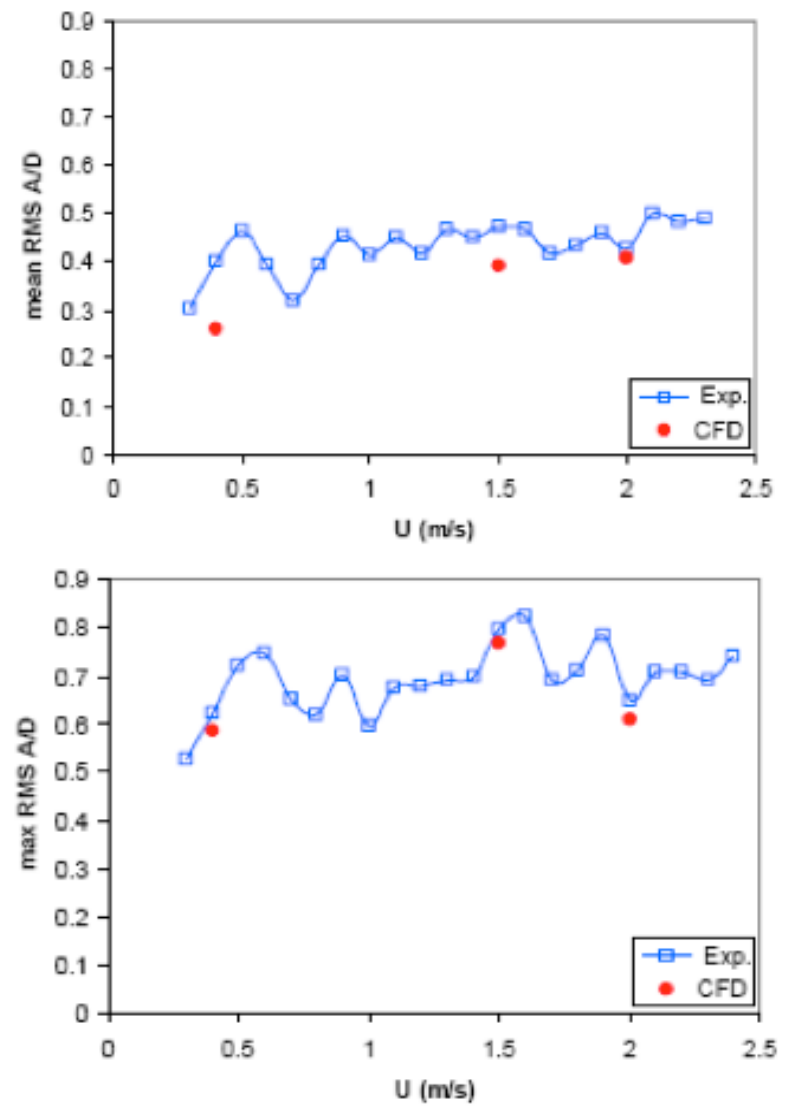

Fig. (17). Mean and max RMS riser response (A/D) values in uniform flow ( $\mathrm{L} / \mathrm{D}=1407)$-- $\mathrm{CFD} v s$. Experiment [68]. 


\section{Experimental Verification}

Modeling a full scale deepwater riser was possible with this analysis (Eq. 24) using a medium-sized computational cluster. Satisfactory experimental validation was obtained from the solution [68] within the limitations, uncertainties and assumptions of the available data and CFD model. An example of the response amplitude/ riser diameter $(A / D)$ versus uniform current flow (equivalent to simulated tow speed) from their simulation is compared with measurements in Fig. (17). The number of nodes and tetra/prism elements used in the CFD analysis for this example were $3.1 \mathrm{M}$ and $10.9 \mathrm{M}$, respectively.

However, some refinements of CFD results are still needed for a successful correlation before confidence in such

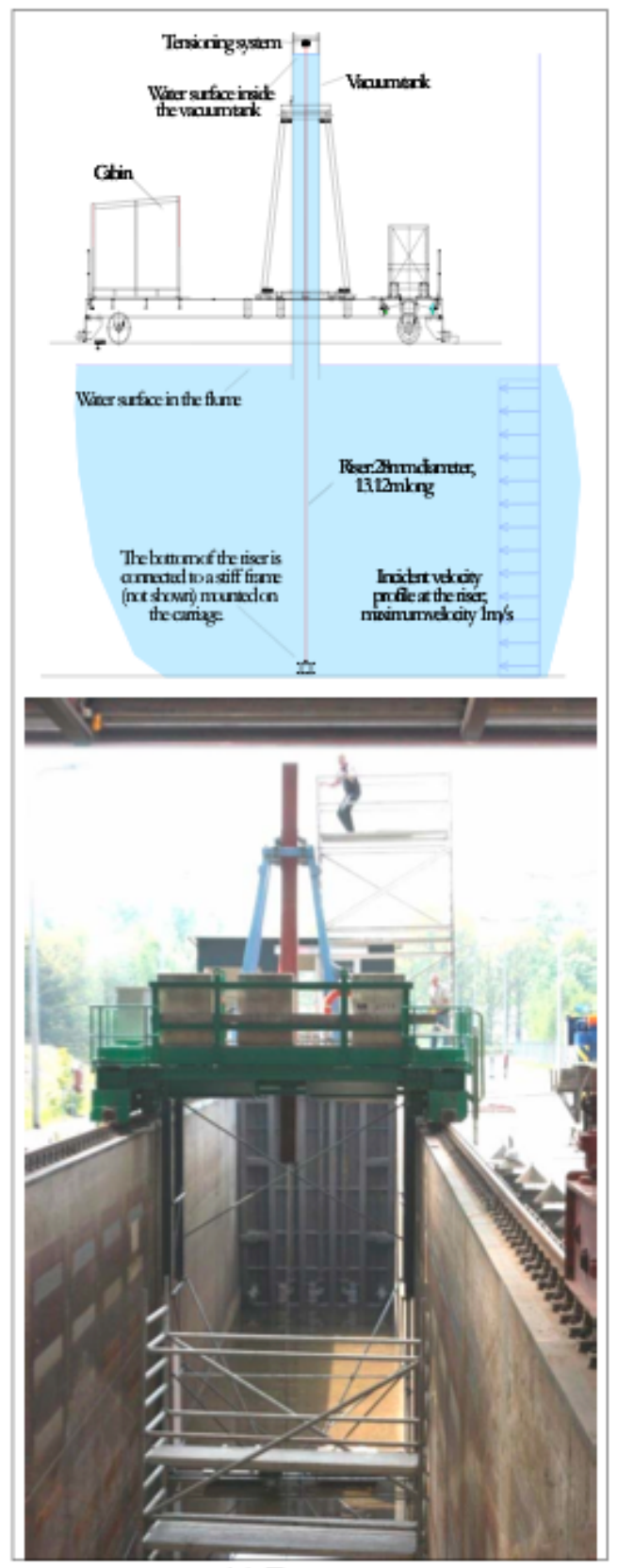

(a) Test setup analysis is established. This is illustrated by the following case. A test was performed (Fig. 18) by towing a long riser model in water and measuring the detailed inline and crossflow response of the riser model [66]. The test setup and input data were supplied to several available software developers, both research type and commercial type for a blind correlation check.

The results from this analysis are given on the right hand side of Fig. (16). It shows the measured inline and cross-flow response compared with results from the various tools. The names of the tools used in this blind test are shown on the left (see the original paper for their identification). It is clear that the correlation is quite varied with different degrees of success without any consistent trends in them. After this comparison test was completed, the results of the physical

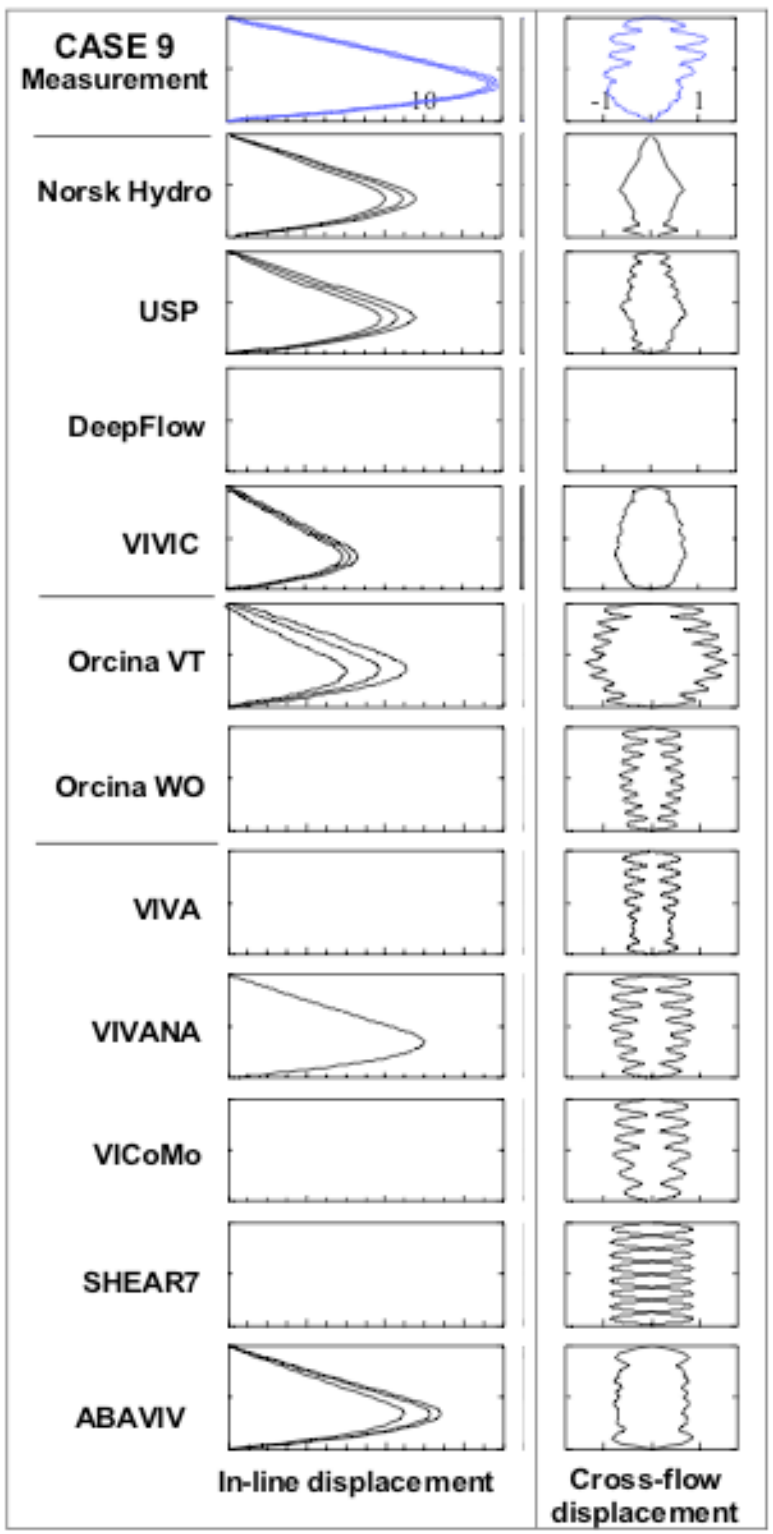

(b) Mode shapes

Fig. (18). Test set up and mode shapes of riser response in steady current [67]. 
tests were made available to the developers/users of the tools for further verification. This latter set of correlations turned out to be much better than the original blind test.

Thus, this type of analysis tool appears to have the capability to predict the results reasonably well, but needs further experience, benchmarking and validation. In order that such validation can be achieved, more accurate benchmark results should be made available. Only then is it possible to make use of such tools in the design or design verification of deepwater risers with confidence.

Among the more complete numerical methods, most of the CFD codes are 2-D assuming parallel planes. They include finite element, finite difference, discrete vortex, vortex-in-cell, and vortex tracking method. A few examples are FSI-Navsim (Norsk Hydro), USP, DeepFlow (IFP), VIVIC (Imperial College), TACC, Orcina VT, and Orcina WO of OrcaFlex. There are also a few large 3-D Commercial Codes currently being adopted for offshore applications, e.g., Fluent, and Acusolve.

Because of the numerous uncertainties in the riser design, a large factor of safety is generally recommended in the fatigue life design of a riser. The guidelines, e.g., API, suggest values of $\mathrm{FOS}=3$, for inspectable riser applications, and FOS $=10-20$, for un-inspectable applications including increased uncertainties, e.g., VIV.

\section{COMPONENT HYDRODYNAMIC COEFFICIENTS}

The biggest difficulty and most challenging in the approximate hybrid numerical/empirical computations of the system response today is an accurate determination of the hydrodynamic coefficients. The hydrodynamic coefficients for the computation of the environmental loads include the inertia, drag and lift coefficients. For the system response calculations, the added mass and damping coefficients are important. The availability of accurate appropriate values applicable to real system components is often rare.

Typical sources of damping [78-84] for a coupled system are: floater drag/viscous damping (which is an important contribution to damping), floater wave drift damping (which is important in the overall structure dynamics), mooring system damping (by far the most important contribution), riser system damping (hydrodynamic type), line internal/material damping (friction forces of individual wires or chain links), damping caused by mooring line on seabed (which generally has a large effect), and thruster damping (only applicable for thruster assisted mooring). The relative contributions of damping from waves, viscous and mooring lines shows that the mooring line has the highest percentage contribution reaching about $80 \%$ overall.

The knowledge of the wave frequency damping of the floater itself is generally adequate. Accurate radiation damping can be computed numerically. The information on the viscous damping is also sufficiently known from numerous model tests and field data. However, the higher order damping, especially in slow drift oscillation of soft-moored vessels or high frequency load on the stiff vertically moored vessels, e.g., TLP, which are critical in the computation of accurate response is difficult to estimate from the available data. For example, the typical damping ratio for a TLP in heave is found from model tests to be about 0.05 percent for both round and square vertical columns, while the same for horizontal pontoons are 0.176 and 0.278 percent (ref: [3, 79, 82, 83]).

An example on the variation of drift damping value with the drift amplitude of a moored system is shown in Fig. (19) for a water depth of $82.5 \mathrm{~m}$, and a drift period of $100 \mathrm{~s}$, having no wave frequency oscillation. Note that damping increased by a factor of 4.5 when the drift amplitude increased from

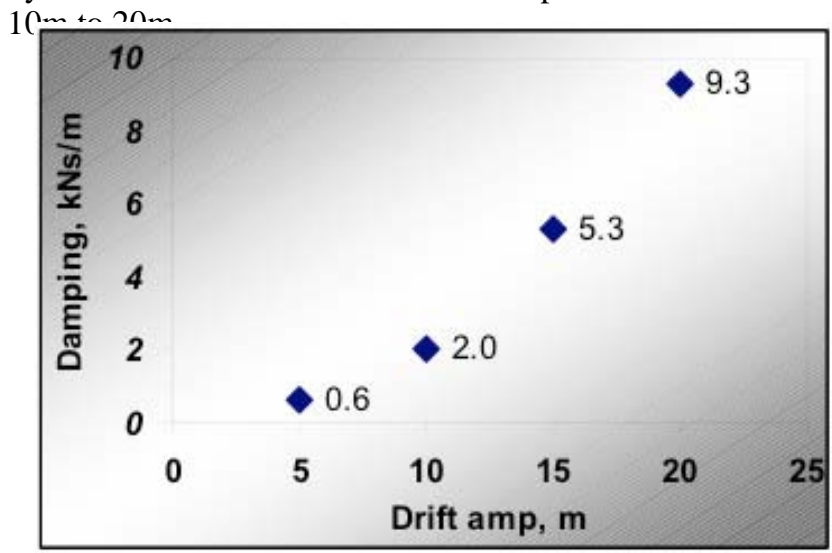

Fig. (19). Chain line damping vs. drift induced top-end amplitude.

The drag coefficients on mooring lines depend on their sectional geometry and magnitudes of current. For example, the drag on chain sections is expected to be much higher than that on wires. If the wires are sheathed, then the drag effect should be small, equivalent to smooth circular cylinders. Typical values of normal drag coefficients on chains are taken as $2.4-2.6$, while that for wires is between $1.2-$ 1.8. The tangential drag component for these components vary from 0.2 to 0.8 . Unfortunately, however, the availability of such data is quite limited and no dependence on the current velocities (or equivalently, the Reynolds number) can be found.

For accurate prediction of the riser response empirically, the knowledge of the hydrodynamic coefficients applicable to the riser geometry is essential. These include the drag and lift coefficients due to waves and current and added mass coefficients. Essentially all the numerical models in riser response development have been concentrated with the current loading. Since the riser geometry is generally close to circular cylinders, it is somewhat easier to determine a reasonable value of the riser hydrodynamic coefficient. However, the values of hydrodynamic coefficients specifically applicable to riser geometry is lacking in the literature.

The drag coefficients for various suppression devices on cylindrical risers vary considerably. The straked risers have the highest value (about 1.4) for $C_{D}$, while it is lowest for fairings. The inline drag coefficients for a fairing mounted riser as functions of Reynolds number is given in Fig. (20) from extensive experiments conducted by Shell Global Solutions. This is the type of information needed for other shapes, which are rarely available.

It has been found that when the cylinder vibrates, such as a riser, the drag coefficients increase in magnitute based on the amplitude of vibration. The drag coefficient of a vibrating cylinder is given by the formula [78]: 


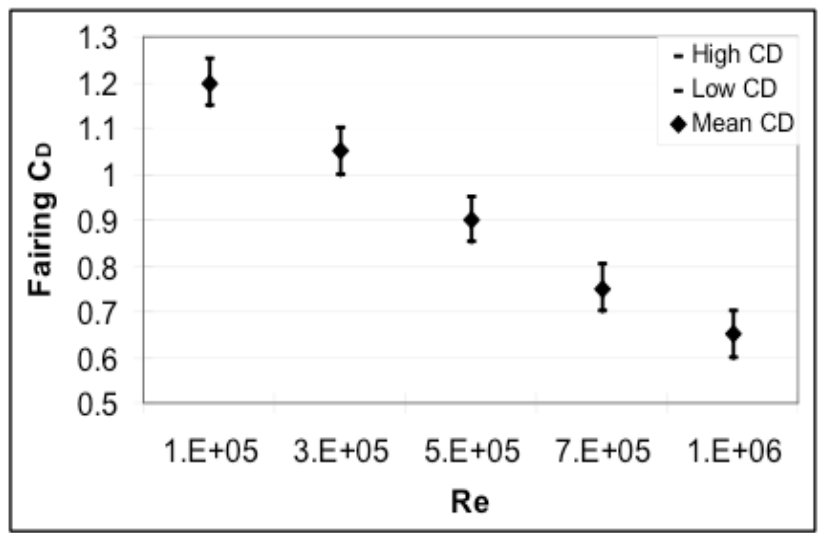

Fig. (20). Drag coefficients for Fairings [Shell Global Solution].

$C_{D}\left(\frac{A}{D}\right)=C_{D(A=0)}\left[1+1.043\left(\frac{2 A_{r m s}}{D}\right)^{0.65}\right]$

in which $C_{D}(A / D)=$ drag coefficient for a vibrating cylinder, $C_{D(A=0)}=$ drag coefficient for a fixed cylinder, $A_{r m s}=$ rms value of cross-flow vibrations, $D=$ hydrodynamic diameter of the cylinder. The plot of this amplification factor vs. the normalized vibration amplitude is shown in Fig. (25). As an example, assume $A_{r m s} / D=0.2$, and $C_{D(A=0)}=1$, then $C_{D}(A / D)=1.57$, which is over a $50 \%$ increase over fixed cylinder.

Risers often appear in a bundle. API [78] Guidelines give the composite blockage factor for a dense structure (composed of many circular members e.g., a riser bundle) in steady current as follows:

$C_{B F}=\left[1+\frac{\sum_{i=1}^{N}\left(C_{D} A\right)_{i}}{4 \bar{A}}\right]^{-1}$

where $N=$ no. in the bundle, $A=$ individual member area, and $\bar{A}=$ overall structure area, projected normal to the flow direction.

The risers mounted with fairings appear to have the highest added mass coefficients of about 1.25 , while the riser with strakes has about the same value as a bare cylinder.

\section{COUPLED ANALYSIS}

Modest efforts have already been made to couple these components. This area is briefly reviewed here. The effects of the appendages become increasingly important, as the structure moves into deeper waters. Thus, their effects in a total system analysis may not be ignored in these cases. However, since a fully coupled time domain analysis of the floater, lines and risers is extremely time consuming with high computational costs, enhanced refinements to the uncoupled system have been proposed, e.g., $[15,19,20]$ which try to balance the accuracy and efficiency [13]. Generally, some level of integration among the floater, mooring lines and risers has been considered in the system analysis [19], even when only uncoupled analysis is used. Such refinements consist in employing enhanced procedures for the determination of the scalar coefficients that are introduced in the floater equations of motion to represent certain important behavior of the mooring lines and risers. A few examples are given below:

- Compute current loads on the mooring lines and include part of it as a 'floater coefficient' at the floater fairlead point.

- Include a composite value of damping due to the motions of mooring lines (and, occasionally, the riser) as a damping coefficient to the hull damping to obtain the overall damping of the system. For example, a scalar damping coefficient may be calibrated from experimental decay test.

- Include a portion of the mass and added mass of the mooring lines to the total mass of the system by introducing an approximate mass coefficient.

However, it has been recognized (refs. [7, 10, 11, 15, 19, $20,27,37,38,48])$ that the most accurate design methodology for floating offshore systems should employ analysis programs based on coupled formulations. Ideally, a coupled analysis should incorporate, in a single code and data structure, a hydrodynamic model for the representation of the vessel, coupled to a 3-D finite-element model for the representation of the hydrodynamic and nonlinear structural dynamic behavior of the mooring lines and risers. The characteristics and advantages of this so-called "fully-coupled" methodology are described by [7]. As already mentioned, one characteristic of this methodology is its excessive computer costs. Therefore, in order to circumvent this problem and to gradually advance towards a fully coupled and integrated design methodology, "hybrid" methodologies and analysis procedures are adopted.

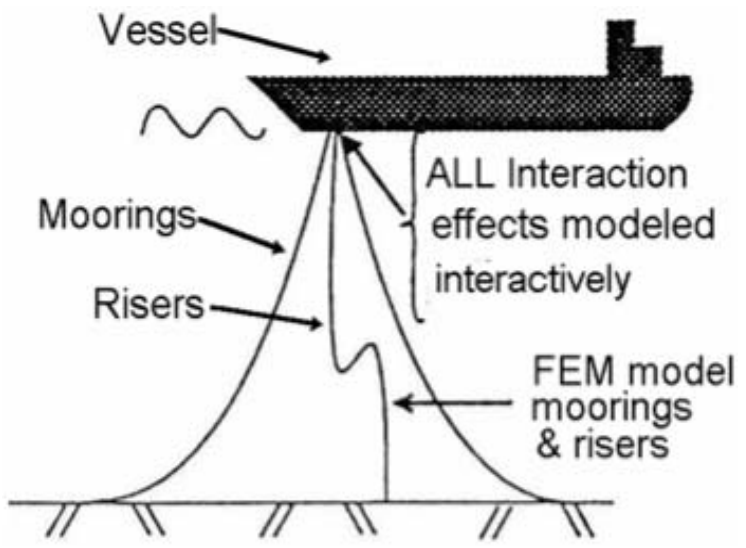

Fig. (21). Schematic for a coupled system analysis (adapted from [15]).

In a coupled analysis, quite often, the linear frequencydependent potential theory is applied for the floater coupled with a 3-D bar/cable finite element for the mooring lines and a 3-D beam element for the riser (Fig. 21). The environmental excitations on the mooring lines and risers are computed by the Morison equation. For the analysis of the individual components, the $6 \mathrm{DOF}$ motions of the floater are introduced as nodal components in the finite-element model [15]. 
In reality, an iterative approach is necessary, since the mooring line/ riser response is coupled to the floater motions. In a complete coupled analysis, two sets of equations of motion are solved, which iterate at a given time step - one for the floater and one for the appendages. In the simulation, the mooring forces in the equation of motion are kept constant over the time step. The 6 DOF motions of the floating vessel is solved at the given time step. During this interval, the equation of motion is integrated with a Runga-Kutta type scheme and the position and velocity of the floater is obtained. The position of the floater is then applied to the top of each of the lines in its dynamic analysis. A finite element or a lumped mass method for the mooring line is applied. For example, to compute the dynamic position of the entire mooring line at each time step in the simulation, a lumped mass method is used (see, for example, [54]), as shown in Fig. 12. In this lumped mass method, the mass of the mooring line is lumped to a finite number of nodes that are connected by linear springs, corresponding to the axial stiffness of the line. Bending stiffness may be required for the riser, but is not taken into account for lines. The current (and wave) loads on the mooring line elements at their nodes are computed. Each mooring line dynamics provide the location and tension at the fairlead point of the lines at this new time step. These new values are imposed to the floater and the 6 DOF of the floater is solved again. Iterations continue until a convergence is reached before proceeding to the next time step. The procedure is similar for the riser.

The difference between the time and frequency domain analysis for a coupled system is illustrated by an example from [13]. It represents a spread moored FPSO using an equivalent four-line mooring system in a water depth of $2000 \mathrm{~m}$. The standard deviation of the line tension (dominated by WF) from the bottom end of a line due to an oblique $15.7 \mathrm{~m}\left(\mathrm{H}_{\mathrm{s}}\right)$ JONSWAP wave is plotted in Fig. (22). While the time domain simulation provided higher tension throughout, the difference in the results from the two analyses is minimal. Thus, in this example, the simpler and more efficient frequency-domain analysis appears to give similar results.

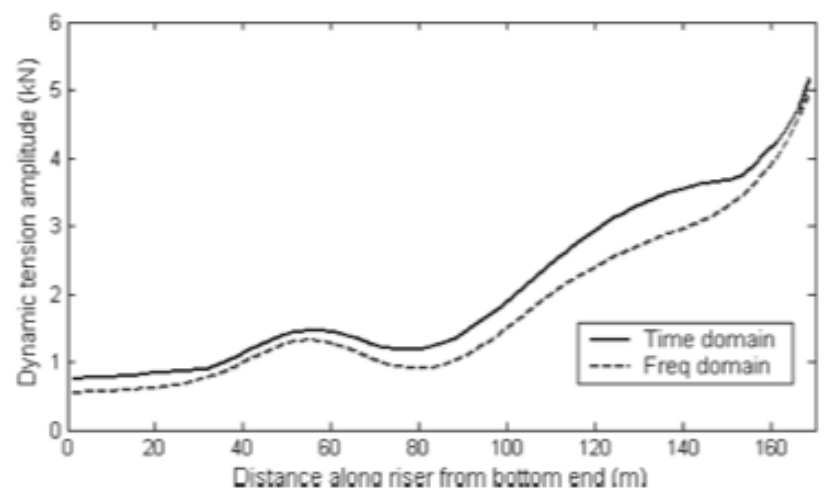

Fig. (22). Time vs. frequency domain line tension for a coupled system analysis [13].

The line tension for the most loaded line is compared by the coupled (C) and uncoupled (U) analysis in Fig. (23) (from [15]). The floater is a turret moored ship with 8 symmetric mooring lines $(\mathrm{M})$ with and without risers $(\mathrm{R})$. The mean and dynamic loads for coupled $(\mathrm{M}$, or $\mathrm{M}+\mathrm{R}$ attached) and uncoupled are shown separately in the results. For the uncoupled analysis (U), the difference between inclusion $(\mathrm{M}+\mathrm{R}$ LF) and exclusion (No LF) of the low frequency loads on the riser and mooring are shown separately. There is some difference evident in the results between the coupled and uncoupled analysis, especially when the risers are included.

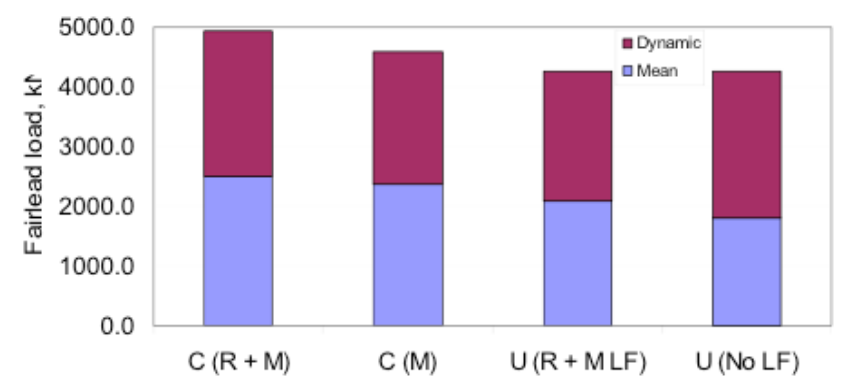

Fig. (23). Mean and dynamic line tensions compared for a coupled (C) vs. uncoupled (U) system (source: [15]).

\section{CONCLUSIONS AND OUTLOOK}

While deepwater development is continuing at a steady pace and considerable progress has been made in the design of offshore structures, several challenges in the analysis of deepwater floating structures remain. The design of large floating systems consisting of floater, mooring lines and risers has, in most part, been carried out using the traditional uncoupled analysis, in which the dynamics of the floater are determined by treating the mooring lines and risers as external stiffness terms. The frequency domain analysis considers the stiffness to be linear, while, if the appropriate nonlinearity is maintained, a time domain method is used. Once the floater dynamics are known, the XYZ floater motions at the appendage connections are applied to the individual mooring line or riser to determine their responses for the design.

For the uncoupled floater, the theory is well-established and several commercially available codes, most of them based on the frequency-domain linear diffraction/radiation theory, are routinely used in the design of such structures. While this method is quite satisfactory, in many cases, the radiation damping is often inadequate for the floater response in the resonance area. Therefore, simple experimental technique (e.g., pluck test) is used to determine the supplemental damping in the overall system. Some quantitative values for them are available, but inadequacy still exists in the availability of these types of data and in their possible inaccuracy.

One other area where further development is being carried out is in the application of NWT. Such methods are capable of handling a variety of problems, not possible with the frequency domain BEM, such as, nonlinear steep waves, wave run-up on floaters, and high wave overtopping on the floater deck. The effects in these specific areas may be a serious design consideration in certain floating offshore systems for which more complete reliable and practical NWT solutions are desirable.

Today's design of risers and mooring lines is generally empirical in nature in which the environmental loading is treated in terms of hydrodynamic coefficients applied to 
these components and a detailed structural analysis of the mooring or riser is carried out based on this loading. Due to lack of knowledge of hydrodynamic data, often a single average coefficient value is used over the entire length of the component, irrespective of its location in the fluid. More systematic analysis should be undertaken to generate more accurate coefficients for these components including appropriate validation.

Moreover, ultradeep water and relatively smaller size of floaters may necessitate the interaction of the appendages and the environmental effects on themselves to be incorporated in a more complete coupled analysis with the floater. This area is still mostly in the developmental stage, even though some limited design verification is currently being carried out by the offshore industry with this type of tool. In this paper, specific recommendations have been made for a systematic procedure into more complete analysis, which is termed the Total System Analysis (TSA).

Progress is being made in the development of more complete and sophisticated numerical analysis for the floating system design. This area needs further development for improved efficiency and accuracy with less dependence on empiricism. Moreover, these techniques need careful validation before their practical application may be warranted in a routine manner by the industry.

\section{REFERENCES}

[1] Chakrabarti SK, Tech Ed. Handbook of Offshore Structures. Elsevier Publication: Oxford, UK.: 2005.

[2] Astrup OC, Nestegard A, Ronoess M, Sodahl N. Coupled analysis strategies for deepwater spar platforms. ISOPE June 2001.

[3] Bunnik THJ, de Boer G, Cozijn JL, der van Cammen J, van Haaften E. Coupled mooring analysis in large scale model tests on a deepwater calm buoy in mild wave conditions. Proceedings of Offshore Mechanics Arctic Engineering Conference OMAE28056 2002.

[4] Chaudhury G. A new method for coupled dynamic analysis of platforms. ISOPE Conference June 2001.

[5] Chitrapu AS, Erketin RC. Time-domain simulation of large amplitude response of floating platforms. Ocean Eng 1995; 22-4 367-85

[6] Connaire A, Kavanagh K, Ahilan RV, Goodwin P. Integrated Mooring Riser Design: Analysis Methodology. Offshore Technology Conference OTC 10810 May 1999.

[7] Correa, FN, Senra, SF, Jacob, BP, Masetti, IQ, Mourelle, MM. Towards the integration of analysis design of mooring systems risers, Parts I \& II: Studies on a Dicas System. Proceedings of Offshore Mechanics Arctic Engineering Conference 2002.

[8] Finn, L, Datta, I, Gupta, H. Coupled Dynamics of Spars in Deep Water. Deep Oil Technology Conference, 2000.

[9] Garrett, D.L, Coupled analysis of floating production systems. Ocean Eng 2005, 32-7: 802-16.

[10] Heurtier JM, Buhan P, LeFontaine E, LeCunff C, Biolley F, Berhault C. Coupled dynamic response of moored FPSO with risers. ISOPE Conference June, 2001.

[11] Kim, MH, Ward, EG, Haring, R. Comparison of numerical models for the capability of hull/mooring/riser coupled dynamic analysis for Spars TLPs in deep ultra-deep waters. ISOPE Conference, June, 2001.

[12] Low, Y.M, Langley, R.S, A simplified model for the study of dynamic coupling effects in deepwater floating structures. Proceedings of Offshore Mechanics Arctic Engineering Conference, Vol. 1A, 2005, 147-156.

[13] Low, Y. M, Langley, R. S, Time frequency domain coupled analysis of deepwater floating production systems. Appl Ocean Res 2006: 28-6.
[14] Ormberg H, Fylling I.J, Larsen K, Sodahl N. Coupled analysis of vessel motions mooring riser system dynamics. Proceedings of Offshore Mechanics Arctic Engineering Conference 1997.

[15] Ormberg H, Larsen K. Coupled analysis of floater motion mooring dynamics for a turret-moored ship. Appl Ocean Res 1998: 20-1-2: 55-67.

[16] Ormberg H, Sodahl N, Steinkjer O. Efficient analysis of mooring systems using de-coupled coupled analysis. Proceedings of Offshore Mechanics Arctic Engineering Conference 1998.

[17] Paulling, J.R. Webster, WC. A Consistent, Large-Amplitude Analysis of the Coupled Response of a TLP Tendon System. Proceedings of Fifth International Offshore Mechanics Arctic Engineering Symposium, ASME, Tokyo, Japan. April 1986: II 126-133.

[18] Ran Z, Kim MH, Zheng W. Coupled dynamic analysis of a moored spar in random waves currents (Time-domain versus frequencydomain analysis). J Offshore Mech Arctic Eng 1999: 121-3: 194200.

[19] Senra SF, Correa FN, Jacob BP, Mourelle MM, Masetti, IQ. Towards the integration of analysis design of mooring systems risers, Part I: Studies on a semisubmersible platform. Proceedings of Offshore Mechanics Arctic Engineering Conference, OMAE200228046, 2002.

[20] Senra SF, Correa FN, Jacob BP, Mourelle MM, Masetti IQ. Towards the integration of analysis design of mooring systems risers, Part II: Studies on a DICAS system. Proceedings of Offshore Mechanics Arctic Engineering Conference; 2002; 1: 291-98.

[21] American Petroleum Institute. Recommended practice for design analysis of station-keeping systems for floating structures. APIRP2SK, Washington DC; 1996.

[22] Chakrabarti SK. Hybrid numerical method for wave multibody interaction (Chapter). Numerical Modeling in Fluid-Structure Interaction. WIT Press Great Britain; 2005.

[23] Chakrabarti SK. Numerical simulation of multiple floating structures with nonlinear constraints. J Offshore Mecha Arctic Eng ASME; May 2002.

[24] De Kat JO, Pauling RJ. The simulation of ship motions capsizing in severe seas. Transactions of the Society of Naval Arch Marine Eng 1989; 97: 139-168.

[25] Langley RS. Second order frequency domain analysis of moored vessels. Applied Ocean Research 1987; 9-1: 7-18.

[26] Menter F, Sharkey P, Yakobov S, Kuntz M. Overview of fluidstructure coupling in ANSYS-CFX. 25th International conference on offshore mechanics arctic engineering, OMAE2006-92145 Hamburg, Germany 2006.

[27] Sen D. Time-domain simulation of motions of large structures in nonlinear waves. Proceedings of 21st International Conference on Offshore Mechanics Arctic Engineering, OMAE2002-28033, Oslo, Norway, 2002.

[28] Beck RF, Cao Y. Lee, TH. Fully nonlinear water wave computations using the desingularized method. Proc. of 6th Int. Conf. on Ship Hydro, 1993, 3-20

[29] Beck RF. Time-domain computation of floating bodies. Applied Ocean Research 1994, 16: 267-82.

[30] Boo SY, Kim CH, Kim MH. A numerical wave tank for nonlinear irregular waves by 3D high-order boundary element method. Int $\mathrm{J}$ Offshore Polar Eng 1994; 4: 265-72.

[31] Celebi. MS, Kim MH, Beck RF. Fully nonlinear 3-D numerical wave tank simulation. Journal of Ship Research 1998;42-1: 33-45.

[32] Dommermuth DG, Yue DKP, Numerical simulations of nonlinear axisymmetric flows with a free surface. J Fluid Mech 1987; 178: 195-219.

[33] Hong SY, Kim MH. Nonlinear wave forces on a stationary vertical cylinder by HOBEM-NWT. Proceedings of 10th International Offshore Polar Engineering Conference Seattle WA 2000; 3: 214-20.

[34] Ikeno M. A numerical model for 3-D floating body motion in nonlinear waves using the BEM. Proc. 10th ISOPE Conf, 2000; 3: 20113. 
[35] Isaacson M, Cheung KF. Time-domain solution for wave-current interactions with a two-dimensional body. Appl Ocean Res 1993; 15: 39-52.

[36] Kashiwagi, M, Nonlinear simulations of wave-induced motions of a floating body by means of MEL method. Proc. of 3rd Int. Conf. on Hydrodynamics, Seoul, 1998.

[37] Kim CH, Clement A, Tanizawa K. Recent research development of numerical wave tanks-a review. Int J Offshore Polar Eng 1999; 9: 241-56.

[38] Kim CH.. Recent progress in numerical wave tank research: a review. 4th Int. Conf. of the Society of Offshore Polar Eng, Osaka, 1995, 9p.

[39] Kim DJ, Kim MH. Wave-current interaction with a large 3D body by THOBEM. J Ship Res 1997; 41-4, 273-85.

[40] Kim MH, Koo WC. 2D fully nonlinear numerical wave tanks. (Chapter 2) Numerical Modeling in Fluid-Structure Interaction (Ed: SK Chakrabarti) WIT Press, Great Britain 2005.

[41] Koo WC, Kim MH. Fully nonlinear waves their kinematics: NWT simulation vs. experiment. Proceedings of 4th International Symposium on Ocean Wave Measurement Analysis, WAVES 2001 ASCE 2001; 2, 1092-1101.

[42] Longuet-Higgins, M, Cokelet ED. The deformation of steep surface waves on water: I. a numerical method of computation. Proceedings of Royal Society of London. 1976; A350: 1-26.

[43] Loots GE, Buchner B. Wave run up as important hydrodynamic issue for gravity based structures. 23rd International Conference on Offshore Mechanics Arctic Engineering, Vancouver British Columbia Canada 2004.

[44] Orlanski JE, A simple boundary condition for unbounded hyperbolic flows. J Comput Phys 1976; 21, 251-69.

[45] Sen D. A time-domain method for wave diffraction radiation of 3D large structures. Proc. 18th International Conference on Offshore Mechanics Arctic Engineering St. John's Canada 1999; 19p.

[46] Stassen Y, Le Boulluec M, Molin B. A high order BEM model for 2D wave tank simulation. Proceedings of 8 th International Offshore Polar Engineering Conference Montreal Canada 1998.

[47] Tanizawa K. The State of the art on numerical wave tank Proceeding of 4th Osaka Colloquium on Seakeeping Performance of Ships, Osaka, Japan, 2000, 95 114.

[48] Tavassoli A, Kim MH. Interactions of fully nonlinear waves with submerged bodies by a 2D viscous NWT. Proceedings of 11 th International Offshore Polar Engineering Conference Stavanger, Norway 2001;. 3, 348-54

[49] Chakrabarti SK, Hydrodynamics of Offshore Structures. Comput Mech Publica: Southampton, UK, 1987.

[50] Tanizawa K, A nonlinear simulation method of 3-D body motions in waves. J Society of Naval Architects of Japan 1995; 178: 17991.

[51] Shirakura, Tanizawa K, Naito. Development of 3-D fully nonlinear numerical wave tank to simulate floating bodies interacting with water waves. Proc. 10th ISOPE Conf: Seattle 2000; 3: 253-62

[52] Chakrabarti SK, Nonlinear Methods in Offshore Engineering. Elsevier Publishers: UK 1990.

[53] Det Norske Veritas. Offshore Standard Position Mooring. DNVOS-E301 June 2001.

[54] Derksen A, Wichers JEW. A discrete element method on a chain turret tanker exposed to survival conditions. Boss Conference 1992.

[55] Garrett DL. Dynamic analysis of slender rods. J Energy Resources Technol 1982; 104-4302-06.

[56] Inoue Y, Surendran S. Dynamics of the interaction of mooring line with the seabed. Proceedings of the 4th International Offshore Polar Engineering Conference: Osaka, Japan, 1994, 317-23.

[57] Low YM, Langley RS. Dynamic analysis of a flexible hanging riser in the time frequency domain. Proceedings of Offshore Mechanics Arctic Engineering Conference 2006.

[58] Kwan CT, Bruen FJ. Mooring Line Dynamics: Comparison of Time Domain, Frequency Domain, Quasi-static Analyses. Proceeding of the 23rd Offshore Technology Conference: Houston TX, May 1991, 95-108.
[59] Ong PA, Pellegrino S. Modelling of seabed interaction in frequency domain analysis of mooring cables. Proceedings of Offshore Mechanics Arctic Engineering Conference OMAE2003374652003.

[60] Wung CC, Litton RW, Mitwally HM, et al. Effect of Soil on Mooring System Dynamics. Proceedings on Offshore Technology Conference OTC007672 May 1995; 1: 301-07.

[61] Bearman PW, Chaplin JR, Fontaine E, et al. Comparison of CFD predictions of multi-mode vortex-induced vibrations of a tension riser with laboratory measurements. ASME Pressure Vessels Piping Division Conference: Vancouver, Canada 2006.

[62] Bearman PW, Huera Huarte FJ, Chaplin JR. The hydrodynamic forces acting on a long flexible circular cylinder responding to VIV, ASME Pressure vessels piping division conference: Vancouver Canada,2006.

[63] Bearman PW, Graham JMR, Lin XW, Meneghini JR. Numerical simulation of flow-induced vibration of a circular cylinder in uniform oscillatory flow. Flow-Induced Vibration, (ed: Bearman, PW).: Rotterdan Balkema, 1995; 231-40.

[64] Chakrabarti SK, Debus, KK, Berkoe,J. Rosendall B. CFD analysis of current-induced loads on large caisson at supercritical Reynolds Number. J Offshore Mechanics Arctic Engineering ASME June 2005.

[65] Chaplin JR, Bearman PW, Huera Huarte FJ, Pattenden RJ. Laboratory measurements of vortex-induced vibrations of a vertical tension riser in a stepped current. J Fluids Struct 2005; 21-1: 3-24.

[66] Chaplin J. Vortex- wake-induced vibrations of deep water risers. Fourth International Conference on Fluid Structure Interaction: Ashurst Southampton UK. 2007.

[67] Chaplin JR, Bearman PW, Cheng Y, et al. Blind predictions of laboratory measurements of vortex-induced vibrations of a tension riser. J Fluids Struct 2005; 2125-40.

[68] Constantinides Y, Oakley OH Jr, Holmes S. CFD high L/D riser modeling study. Proceedings of the 26th International Conference on Offshore Mechanics Arctic Engineering OMAE2007-29151: San Diego CA, June, 2007.

[69] Chung J, Hulbert GM. A time integration algorithm for structural dynamics with improved numerical dissipation: the generalized method. J Appl Mech 1993; 60: 371-75

[70] Fumes GK, Hassanein T, Halse KH, Eriksen M. A field study of flow induced vibrations on a deepwater drilling riser. Proceedings of the Offshore Technology Conference \# 8702, 1998; 199-208.

[71] Ghadimi R. A simple efficient algorithm for the static dynamic analysis of flexible marine risers. Computers Structures, 1988; 294: 541-44.

[72] Huera Huarte, F. J, Bearman, P. W, Chaplin, J. R, On the force distribution along the axis of a flexible circular cylinder undergoing multi-mode vortex-induced vibrations. J Fluids Struct, Vol. 22, No. 6-7, 2006, 897-903.

[73] Hughes TJR. Recent progress in the development understanding of SUPG methods with special reference to the compressible Euler Navier-Stokes equations. Int J Numer Methods Heat Fluid 1987, 1261-75.

[74] Larsen CM, Baarholm GS, Lie H. Influence from helical strakes on vortex induced vibrations static deflection of drilling risers. Proceedings of 24th International Conference on Offshore Mechanics Arctic Engineering OMAE2005-67192, Halkidiki Greece; June 2005.

[75] Raman-Nair W, Baddour R E, Three-dimensional dynamics of a flexible marine riser undergoing large elastic deformations. Multibody System Dynamics, Vol. 10, No. 3, 2003, 393-423.

[76] Spalart PR, Allmaras SR. A one-equation turbulence model for aerodynamics flows. AIAA \#92-0439: Reno NV 1992.

[77] Spalart PR, Jou WH, Strelets M, Allmaras SR. Comments on the feasibility of LES for wings, on hybrid RANS/LES Approach. Advances in DNS/LES: Greyden Press Aug. 1997.

[78] American Petroleum Institute. Recommended practice for planning, designing constructing fixed offshore platforms. API-RP2A, 21st Edition: Washington DC Dec. 2000 
[79] Chakrabarti SK, Hanna SY. High frequency hydrodynamic damping of a TLP leg. Proceedings of the Offshore Mechanics Arctic Engineering Symposium: Stavanger Norway, June 1991; I-A: 14752.

[80] Garrett DL, Gordon RB, Chappell JF. Mooring - riser-induced damping in fatigue seastates. Proceedings of Offshore Mechanics Arctic Engineering Conference 2002; 793-9.

[81] Huang S, Clelland D, Day, S. Drag reduction of deepwater risers by the use of helical grooves. Proceedings of the 26th International Conference on Offshore Mechanics Arctic Engineering OMAE2007-29454: San Diego CA June, 2007.
[82] Huse E, Utnes T. Springing damping of Tension Leg Platforms. Proceedings of 26th Offshore Technology Conference \#7446 1994; 259-67.

[83] Huse E. Resonant heave damping of Tension Leg Platforms. Proceedings of 22nd Offshore Technology Conference \#6317 1990; 431-6.

[84] Wichers JEW, Devlin PV. Effect of coupling of mooring lines risers on the design values for a turret moored FPSO in deep water of the Gulf of Mexico. ISOPE June 2001.

(C) Subrata Chakrabarti; Licensee Bentham Open.

This is an open access article distributed under the terms of the Creative Commons Attribution License (http://creativecommons.org/licenses/by/2.5/), which permits unrestrictive use, distribution, and reproduction in any medium, provided the original work is properly cited. 